\title{
Nanomechanics of the Cartilage Extracellular Matrix
}

\section{Lin Han, ${ }^{1}$ Alan J. Grodzinsky, ${ }^{2,3,4}$ and Christine Ortiz ${ }^{1}$}

${ }^{1}$ Department of Materials Science and Engineering, ${ }^{2}$ Department of Electrical Engineering and Computer Science, ${ }^{3}$ Department of Mechanical Engineering, and ${ }^{4}$ Department of Biological Engineering, Massachusetts Institute of Technology, Cambridge, Massachusetts 02139; email: cortiz@mit.edu

Annu. Rev. Mater. Res. 2011. 41:133-68

The Annual Review of Materials Research is online at matsci.annualreviews.org

This article's doi:

10.1146/annurev-matsci-062910-100431

Copyright (C) 2011 by Annual Reviews. All rights reserved

$1531-7331 / 11 / 0804-0133 \$ 20.00$

\section{Keywords}

tissue engineering, aggrecan

\begin{abstract}
Cartilage is a hydrated biomacromolecular fiber composite located at the ends of long bones that enables proper joint lubrication, articulation, loading, and energy dissipation. Degradation of extracellular matrix molecular components and changes in their nanoscale structure greatly influence the macroscale behavior of the tissue and result in dysfunction with age, injury, and diseases such as osteoarthritis. Here, the application of the field of nanomechanics to cartilage is reviewed. Nanomechanics involves the measurement and prediction of nanoscale forces and displacements, intra- and intermolecular interactions, spatially varying mechanical properties, and other mechanical phenomena existing at small length scales. Experimental nanomechanics and theoretical nanomechanics have been applied to cartilage at varying levels of material complexity, e.g., nanoscale properties of intact tissue, the matrix associated with single cells, biomimetic molecular assemblies, and individual extracellular matrix biomolecules (such as aggrecan, collagen, and hyaluronan). These studies have contributed to establishing a fundamental mechanism-based understanding of native and engineered cartilage tissue function, quality, and pathology.
\end{abstract}




\section{INTRODUCTION}

The field of nanomechanics involves the measurement and prediction of nanoscale forces and displacements, intra- and intermolecular interactions, spatially varying mechanical properties, local constitutive laws, molecular-level structure-property relationships, and mechanical phenomena that exist at small length scales within and between materials (1-6). In recent years, experimental and theoretical nanomechanics methods have begun to be applied to the field of musculoskeletal tissues at varying levels of material complexity, e.g., nanoscale properties of intact tissue, the matrix associated with single cells, biomimetic molecular assemblies, and individual biomolecules. These data, in conjunction with biochemical and structural analysis methods, have contributed to a fundamental, mechanism-based understanding of musculoskeletal tissue function, quality, and pathology.

Quantification of nanomechanical properties associated with tissue development and pathology has the potential to differentiate tissue types, enable early-stage diagnosis of diseases, document disease progression, and assess and optimize treatment interventions, all at unparalled resolutions (7-10). Furthermore, the application of nanomechanics to the fields of regenerative medicine and tissue engineering will contribute to advancements toward tissue repair and/or replacement for people afflicted with debilitating ailments, such as osteoarthritis (11-13). Medical applications of nanotechnology are in their earliest stages and have yet to advance to significant clinical use in vivo $(8-10,14)$. There is thus a gap between the nanometer length scale of biological processes that are fundamental to musculoskeletal health and disease and the much larger length scale associated with conventional diagnostic and therapeutic approaches. This length scale mismatch is one important reason why traditional tissue engineering approaches in the musculoskeletal field, after 15 years, do not yet have wide-ranging clinical or commercial impact $(15,16)$.

\subsection{Cartilage Tissue Function and Properties}

This review focuses on one particular musculoskeletal tissue-cartilage—as a model system. Cartilage is a biomacromolecular fiber composite material located at the ends of long bones that enables proper joint lubrication, articulation, and loading (Figure 1a). During joint motion, cartilage sustains a complex combination of compressive, shear, and tensile stresses up to $\sim 20 \mathrm{MPa}(18,19)$ and can withstand compressive strains of 10-40\% (20). Cartilage also exhibits excellent lubrication properties and wear resistance, with friction coefficients reported between $\sim 0.0005$ and 0.04 in the presence of synovial fluid (21), a feature discussed extensively in the literature and attributed

\section{Figure 1}

Schematic illustration of the molecular constituents in cartilage and their arrangement into large multimolecular assemblies.

(a) Macroscale comparison of normal (healthy) and osteoarthritic human cartilage tissue; courtesy of Drs. D. Chai \& C. Wheeler.

(b) Cross-sectional schematic of cartilage tissue showing a depth-dependent zone and gradient in cell size, shape, and collagen network morphology. The cells are flattened near the surface (superficial zone) and become larger and rounder with increasing depth in the middle and deep zones. The tidemark is the boundary between the nonmineralized and the mineralized (calcified) cartilage. In addition, the cartilage extracellular matrix is organized into pericellular, territorial, and interterritorial matrices, each of which is present at increasing distance from the chondrocyte cell surface. Panel $b$ adapted with permission from Reference 34. (c) Matrix molecular composition and organization in the different extracellular regions. At the cell surface, many receptors interact with specific matrix molecules as well as with soluble proteins (e.g., growth factors). Matrix molecules in this pericellular zone are also connected to molecules in the territorial region. Panel $c$ adapted with permission from Reference 35. (d) Nanostructures of different cartilage molecular constituents via tapping-mode AFM imaging. Type II collagen fibrils from proteoglycan-digested calf knee cartilage surface (amplitude image), single aggrecan and hyaluronan molecules, and aggrecan aggregates composed of fetal bovine aggrecan noncovalently bound to hyaluronan, which is further stabilized by the small globular link protein (beight images). Panel $d$ reproduced with permission from Reference 36 and courtesy of Dr. H.-Y. Lee. 
a

Normal cartilage

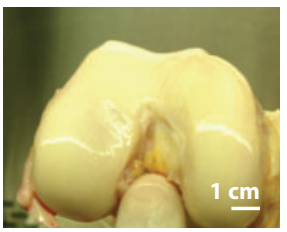

Osteoarthritic cartilage

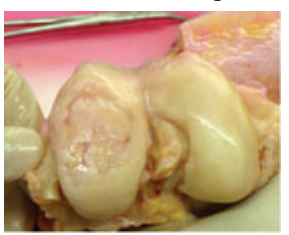

C Cartilage matrix

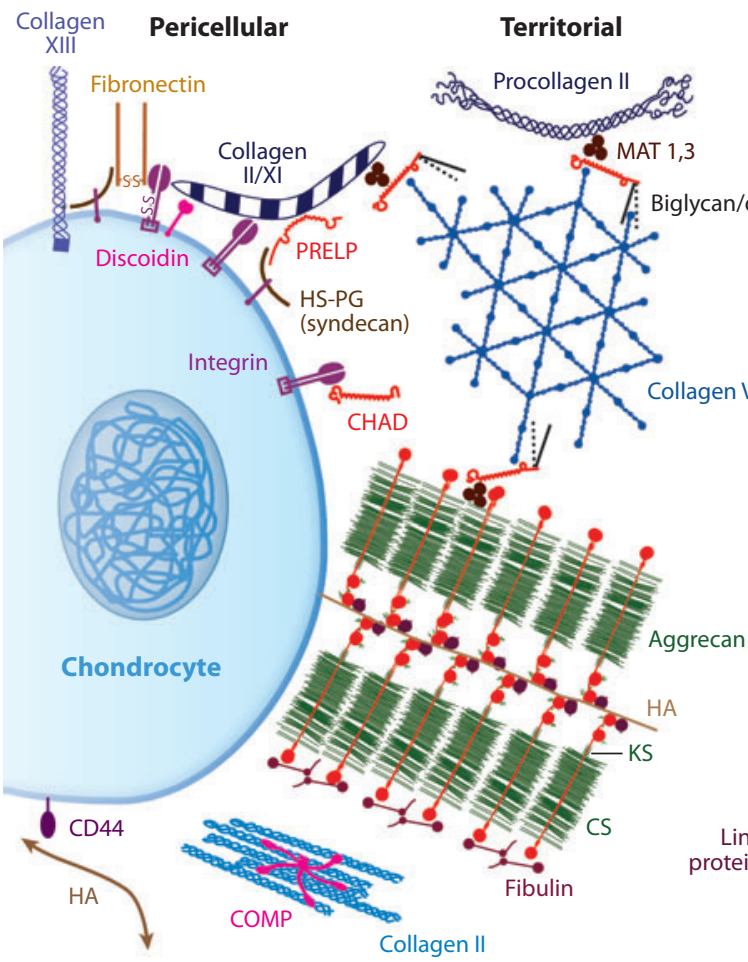

d
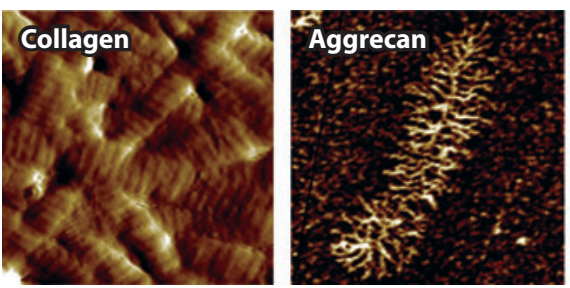

b

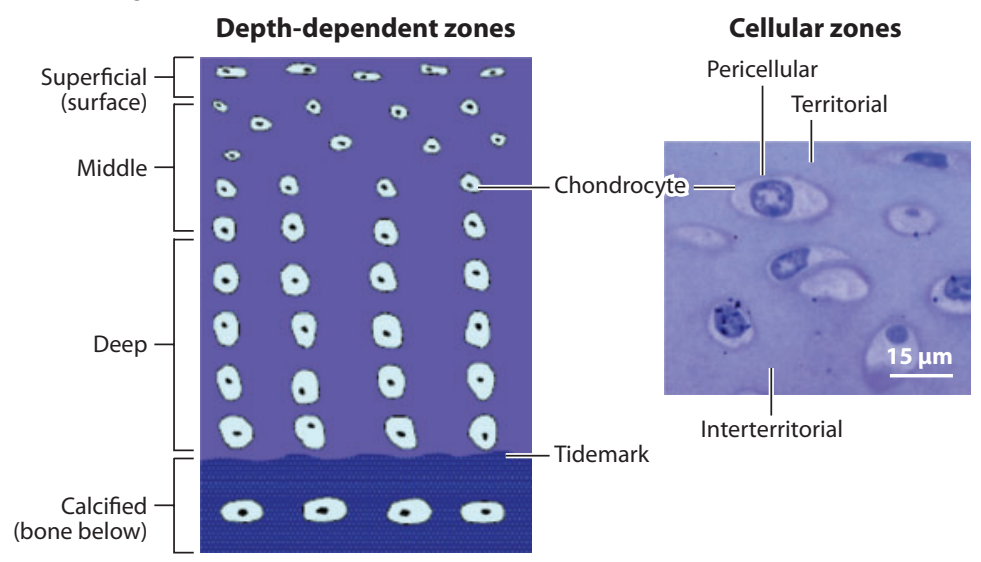

Interterritorial

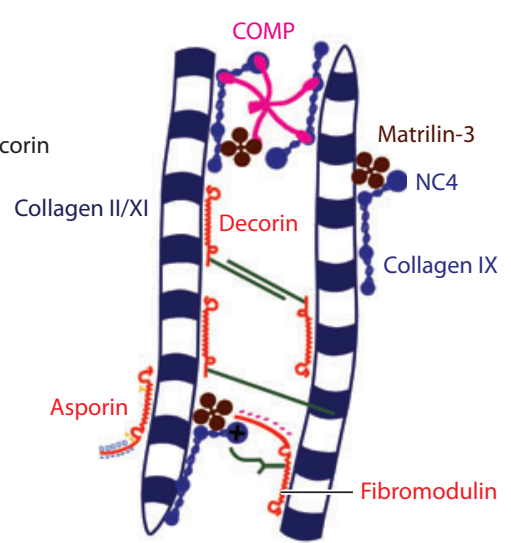

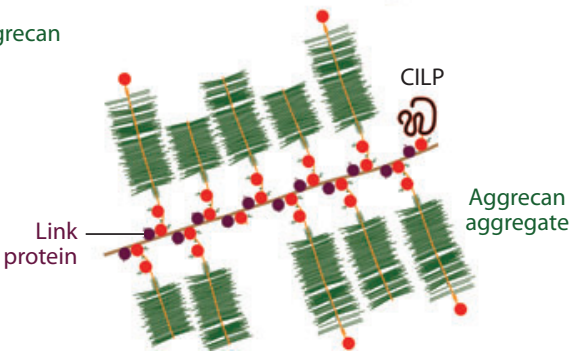
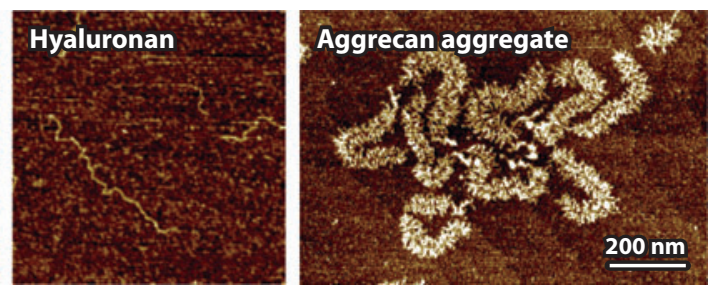
to interstitial pressurization $(168,169)$ and to boundary lubrication by charged molecules $(153$, 170). The biomechanical function of cartilage is attributable to the tissue's macromolecular extracellular matrix (ECM) (18). Degradation of the ECM molecular components and changes in their nanoscale structures greatly influence the macroscale behavior of the tissue and can result in loss of function with age and disease (Figure 1a) (22). To understand the function of cartilage at the tissue level, cartilage mechanical properties have been studied in detail via many different macroscopic loading configurations, e.g., confined (23) and unconfined (24) compression, pure and simple shear $(25,26)$, osmotic swelling (27), and indentation $(28,29)$. These experiments have demonstrated the critically important equilibrium elastic deformation range of the tissue, as well as the range of nonlinear equilibrium behavior. Cartilage also exhibits unique time-dependent properties associated with flow-independent intrinsic macromolecular viscoelasticity (30) and fluid flow-dependent poroelasticity (31). Both electrostatic and nonelectrostatic interactions contribute to these material properties.

\subsection{Molecular Structure, Composition, and Function of Cartilage}

As Table 1 shows, cartilage is a hydrated, avascular tissue composed of $\sim 65-75 \% \mathrm{w} / \mathrm{w}$ water and ECM, as well as cartilage cells (Figure $\mathbf{1 b}$ ). The cartilage cells, or chondrocytes, are responsible for the synthesis, maintenance, and turnover of the ECM components. Despite their critical biological function, chondrocytes make up only 3-5\% of the volume of adult articular cartilage (32). Because the stiffness of the chondrocyte is two to three orders of magnitude less than that of the ECM, these cells do not contribute significantly to the bulk mechanical properties of the tissue (33).

The load-bearing capability of cartilage is sustained primarily by two ECM components: the fibrillar collagen network and the highly negatively charged proteoglycan aggrecan, which account for $\sim 20-30 \%$ and $\sim 10 \%$ of cartilage (w/w), respectively (Figure 1c) (18). The heteropolymeric collagen fibrils consist of types II, IX, and XI: Central filaments of type XI collagen are surrounded by type II fibrils ( $\sim 90 \%$ of the collagen mass) and an outer layer of type IX fibrils, thought to facilitate interactions with other ECM constituents (37). Intra- and intermolecular cross-links contribute to the mechanical properties of collagen fibrils (35). In vivo, collagen fibrils are $\sim 30-80 \mathrm{~nm}$ in diameter and are spaced $\sim 100 \mathrm{~nm}$ apart (Figure $\mathbf{1} \boldsymbol{d}$ ), with chondrocytes, densely packed proteoglycans, and other ECM molecules entrapped within the network (38). Given the intrinsically high molecular stiffness of tropocollagen and the highly oriented (anisotropic) structure of the crosslinked fibrils, the collagen network is primarily responsible for the cartilage tensile properties (18).

In addition, the cartilage matrix contains a superfamily of heavily glycosylated proteoglycans, which are molecules that consist of a protein peptide core that is substituted with one or more glycosaminoglycan (GAG) chains (linear polysaccharide chains). The most abundant proteoglycan, aggrecan, is a highly negatively charged, brush-like proteoglycan macromolecule (39). Along its core protein backbone (contour length $L_{c} \sim 400 \mathrm{~nm}$ ) exist densely packed, negatively charged GAGs, including chondroitin sulfate glycosaminoglycan (CS-GAG; $L_{c} \sim 40 \mathrm{~nm}$ ) and shorter keratin sulfate glycosaminoglycan (KS-GAG) side chains (40). In vivo, aggrecan molecules are entrapped within the porous collagen network. They are bound noncovalently to molecules of another long-chain GAG, hyaluronan (also known as hyaluronic acid or HA); this binding is further stabilized by link protein [a small globular protein synthesized by chondrocytes independently and simultaneously with aggrecan and HA (41)]. The spacing between adjacent aggrecan molecules along the HA chain is $\sim 20-50 \mathrm{~nm}$, and these molecules thereby form large aggrecan aggregates (Figure $1 \boldsymbol{d}$ ). The resulting aggrecan-water gel contributes to a number of important characteristics of cartilage, such as its osmotic swelling pressure (42), hydraulic permeability (18), and resistance to compressive $(44,45)$ and shear $(26,46)$ deformation. In the early stages of 
Table 1 Summary of the major biomechanically functional molecular components of cartilage extracellular matrix. See References 32 and 35 for detailed reviews

\begin{tabular}{|c|c|c|c|c|}
\hline $\begin{array}{c}\text { Molecular } \\
\text { component }\end{array}$ & Structure & Molecular weight & Location & Mechanical function \\
\hline Collagen fibrils & $\begin{array}{l}\text { Fibrillar network of the } \\
\text { interterritorial matrix } \\
\text { (composed of collagen types } \\
\text { II/IX/XI): molecular } \\
\text { contour length } L_{c} \text { of type II } \\
\text { collagen } \sim 300 \mathrm{~nm} \text {, fibril } \\
\text { diameter } \sim 30-80 \mathrm{~nm}, \\
\text { interfibrillar spacing } \\
\sim 100 \mathrm{~nm}(38)\end{array}$ & $\begin{array}{l}\text { 0.4 MDa (single } \\
\text { triple-helical } \\
\text { molecule complex) } \\
\text { (National Center for } \\
\text { Biotechnology } \\
\text { Information) }\end{array}$ & $\begin{array}{l}\text { Extracellular } \\
\text { matrix (ECM) } \\
\text { (territorial and } \\
\text { interterritorial } \\
\text { matrix) (18) }\end{array}$ & Tensile strength (18) \\
\hline $\begin{array}{l}\text { Aggrecan (the most } \\
\text { abundant } \\
\text { proteoglycan) }\end{array}$ & $\begin{array}{l}\text { Brush-like molecule with } \\
\text { glycosaminoglycan (GAG) } \\
\text { side chains (protein core } L_{c} \\
\sim 400 \mathrm{~nm} \text {, chondroitin } \\
\text { sulfate and keratin sulfate } \\
\text { GAG side chains; see } \\
\text { below); noncovalently binds } \\
\text { to hyaluronan, stabilized by } \\
\text { link protein, to form the } \\
\text { aggrecan aggregate (41) }\end{array}$ & $\begin{array}{l}\sim 2-3 \mathrm{MDa} \\
(\sim 200-300 \mathrm{MDa} \text { for } \\
\text { the aggregate })(70)\end{array}$ & \multirow[t]{3}{*}{$\begin{array}{l}\text { ECM (pericellular, } \\
\text { territorial, and } \\
\text { interterritorial } \\
\text { spaces); content } \\
\text { varies with depth } \\
\text { in tissue (18) }\end{array}$} & \multirow[t]{3}{*}{$\begin{array}{l}\text { Osmotic (electrostatic) } \\
\text { swelling pressure (42) } \\
\text { and hydraulic } \\
\text { permeability (18), } \\
\text { which together confer } \\
\text { static and dynamic } \\
\text { compressive }(44,45) \\
\text { and shear }(26,46) \\
\text { strength }\end{array}$} \\
\hline $\begin{array}{l}\text { Chondroitin sulfate } \\
\text { glycosaminoglycan }\end{array}$ & $\begin{array}{r}\text { Linear GAG molecule } \\
\left(L_{c} \sim 40-50 \mathrm{~nm}\right)(40)\end{array}$ & $\sim 0.02-0.03 \mathrm{MDa}(40)$ & & \\
\hline $\begin{array}{l}\text { Keratin sulfate } \\
\text { glycosaminoglycan }\end{array}$ & $\begin{array}{l}\text { Linear GAG molecule } \\
\left(L_{c} \sim 10 \mathrm{~nm}\right)(40)\end{array}$ & $\begin{array}{l}\sim 0.005-0.01 \mathrm{MDa} \\
(158)\end{array}$ & & \\
\hline Hyaluronan & $\begin{array}{l}\text { Linear GAG molecule } \\
\left(L_{c} \sim 1-7 \mu \mathrm{m}\right)(50)\end{array}$ & $\sim 0.5-6 \mathrm{MDa}(50,70)$ & $\begin{array}{l}\text { Within ECM as } \\
\text { part of aggrecan } \\
\text { aggregate (18); in } \\
\text { synovial fluid as a } \\
\text { lubricant (52) }\end{array}$ & $\begin{array}{l}\text { Formation of aggregate } \\
(41), \text { lubrication } \\
(53-55)\end{array}$ \\
\hline Lubricin (PRG4) & $\begin{array}{l}\text { Mucin glycoprotein: core } \\
\text { protein with } \mathrm{N} \text { - and } \\
\text { O-linked oligosaccharide } \\
\text { side chains (core protein } \\
L_{c} \sim 200 \mathrm{~nm} \text {, side chain } \\
\left.L_{c} \sim 0.5-1 \mathrm{~nm}\right)(58,59)\end{array}$ & $\begin{array}{l}\sim 0.230-0.30 \mathrm{MDa} \\
\quad(57)\end{array}$ & $\begin{array}{l}\text { In cartilage } \\
\text { superficial zone } \\
\text { and in synovial } \\
\text { fluid (57) }\end{array}$ & Lubrication (61) \\
\hline
\end{tabular}

osteoarthritis, aggrecan is one of the first components to be degraded and released from cartilage due to increases in the concentration and activity of enzymes termed aggrecanases $(47,48)$. Aggrecanases are synthesized by chondrocytes in cartilage and by cells in other nearby joint tissues. They can cleave the covalent links along the core protein amino acid sequence and break aggrecan into smaller fragments, which then diffuse out of cartilage. The resulting degradation and loss of aggrecan cause instantaneous changes in cartilage biomechanical function, as marked by a decrease in load-bearing capacity. These changes lead to further damage upon continuous joint loading (49). The mechanical function of aggrecan and the effects of aggrecanase cleavage and subsequent loss of aggrecan on cartilage mechanical properties can be studied ex vivo using organ culture explants of native cartilage that can be harvested from animal or human joints and maintained in culture medium for weeks $(22,24,44,45)$. 
HA is a long, linear, negatively charged GAG chain (Figure 1 $\mathbf{d}$ ) (50). In vivo, HA can bind up to $\sim 100$ aggrecan monomers to form the aggrecan aggregate. In addition, HA binds to the surface of many cells by the CD44 cell receptor; this HA binding can inhibit cell-cell adhesion and facilitate cell migration (51). HA is also present in abundance in the viscous, lubricating synovial fluid between the cartilage tissue at the two ends of a joint (52), and hence it may play a role in cartilage biolubrication. Past studies have suggested possible roles of HA, including its involvement in synovial fluid viscosity (53) and hydrodynamic and boundary lubrication (54) and as a transporter for lubricating phospholipids (55). However, to what extent HA contributes to cartilage biolubrication remains unclear (56).

Lubricin, also known as superficial zone protein or PRG4, is a linear glycoprotein present in the synovial fluid at $\sim 250 \mu \mathrm{g} \mathrm{ml}^{-1}$ (57). Lubricin has a core protein with $L_{c} \sim 200 \mathrm{~nm}(58,59)$, and its central mucin-like domain is heavily packed with negatively charged O-linked oligosaccharides (60). Its two hydrophobic globular domain ends (which are positively charged) play a role in cell-cell and cell-ECM interactions (57). Lubricin is thought to play a major role in cartilage biolubrication and wear protection (61). At the tissue level, removal of lubricin from the cartilage surface significantly increases the cartilage friction coefficient (62).

Other ECM molecular components of cartilage play important functions in tissue assembly and integrity. These molecules function as cross-linkers for the formation of the interconnected collagen network, such as the families of matrilins $(63,64)$, small leucine-rich proteins (SLRPs) [including decorin, asporin, fibromodulin, lumican, keratocan, and osteoadherin (6567)], and thrombospondins (68), as illustrated schematically in Figure 1 $\boldsymbol{c}$ (35). Other SLRPs [including chondroadherin, osteoadherin, and proline/arginine-rich end leucine-rich repeat protein (PRELP)] can bind to chondrocyte cell surface receptors and are thought to facilitate cell signal transduction, bridging between the cell and the ECM, and cell migration (69).

\subsection{Open Questions in Cartilage Research}

Despite decades of research in this area, there are still outstanding questions that necessitate a more fundamental molecular perspective of tissue-level biomechanical function. For example:

- Although collagen and aggrecan dominate tissue mechanical behavior, which of the dozens of other ECM proteins, proteoglycans, and glycoproteins are critical for the proper assembly of the collagen fibrillar network, the assembly of aggrecan aggregates, and the molecular linkages between collagen fibrils and aggrecan?

- What is the role of GAG contour length and inter-GAG spacing in optimizing GAGGAG electrostatic and nonelectrostatic interactions that underlie macroscopic compressive stiffness?

- What are the biophysical and molecular mechanisms that contribute to flow-independent viscoelastic behavior? Is there more than one regime (mechanism) of viscoelastic behavior?

- Does poroelastic behavior manifest itself only at the macroscale, or do flow-dependent kinetics occur at the nanoscale dimensions of the collagen fibril, the aggrecan macromolecule, or the pore spaces between neighboring GAG chains?

It is the hope that advances in nanomechanics of the ECM will ultimately provide answers to such questions. In this review, we cover recent advances in nanomechanical studies of cartilage and its ECM constituents. Section 2 reviews nanomechanical methods utilized for these studies, including instrumented (depth-sensing) indentation, atomic force microscopy (AFM)-based spectroscopy, surface force apparatus (SFA), and optical tweezers. Section 3 describes recent advances in nanomechanical studies of native cartilage tissue. Section 4 reviews studies of molecular assemblies of major 
cartilage ECM components, including CS-GAGs, aggrecan, hyaluronan, and lubricin. Section 5 focuses on studies of single-molecule (mechanical) properties of cartilage ECM molecular constituents. The knowledge obtained from these studies provides high-resolution information on the molecular origins of cartilage tissue function and contributes insights into strategies for cartilage tissue engineering and repair.

\section{NANOMECHANICS METHODS USED TO STUDY CARTILAGE}

Traditionally, cartilage has been treated as a macroscopic continuum (either homogeneous or having depth-dependent material properties). Recent molecular-level theoretical models (71-74) have shown the potential to link ECM molecular interactions to tissue-level mechanical properties. Hence, to fully understand cartilage mechanical function and dysfunction, it is essential to probe the tissue at length scales on the same order as that of its zonal and territorial regions (microscale) and its ECM macromolecules (nanoscale). Deformations that take place at the length scale of aggrecan molecules and their constituent GAGs can affect local fixed charge density (71), hydraulic permeability (75), streaming potential (76), and other biophysical mediators of chondrocyte cell signaling and mechanotransduction (77). Nanomechanical methods have the advantage of probing spatial variations in cartilage mechanical properties at high resolutions. Such variations are directly related to tissue function in different zonal or territorial regions at the microscale or to the properties of different molecular constituents at the nanoscale. These methods also enable the investigation of properties of the individual cartilage ECM constituents (e.g., collagen, aggrecan, HA, lubricin) in the form of molecular assemblies or isolated single molecules, both of which are critical for understanding cartilage properties from a molecular perspective. There have been extensive reviews on the fundamentals and technical details of nanomechanical instrumentation, experimental procedures, and data analysis. Here, we summarize topics specifically relevant to cartilage that include use of the following nanomechanical methods: AFM-based indentation (78), high-resolution force spectroscopy (HRFS) (79), single-molecule force spectroscopy (SMFS) (6) and lateral force microscopy (LFM) (80), instrumented (depth-sensing) indentation (81), optical tweezers (6), and SFA (82, 83). These methods are summarized in Table 2.

\subsection{Nanoindentation}

Instrumented (depth-sensing) nanoindentation and AFM-based nanoindentation have been employed to measure penetration force versus indentation depth on cartilage specimens. A number of indenter geometries have been employed, including spherical, conical, pyramidal, and Berkovich. One important parameter extracted from nanoindentation data is the indentation modulus of the sample, $E_{\text {ind }}$, which represents the local resistance to penetration during elastic multiaxial loading. One approach to determining $E_{\text {ind }}$ is the Oliver-Pharr method (84), which is based on a continuum, isotropic, homogeneous elastic contact model to determine the reduced modulus, $E_{r}$, from the unloading portion of the force-depth data:

$$
S=\frac{d F}{d D}=\frac{2}{\sqrt{\pi}} E_{r} \sqrt{A},
$$

where $S$ is the slope of the initial portion of the unloading force $(F)$-depth $(D)$ curve and $A$ is the projected contact area of the indenter. $E_{\text {ind }}$ of the tested specimen is then calculated using

$$
\frac{1}{E_{r}}=\frac{\left(1-v^{2}\right)}{E_{\text {ind }}}+\frac{\left(1-v_{i}^{2}\right)}{E_{i}},
$$


Table 2 Nanomechanics methods applied to cartilage tissue $\mathrm{e}^{\mathrm{a}}$

\begin{tabular}{|c|c|c|c|c|c|}
\hline Method & Load-depth range & Geometry & $\begin{array}{c}\text { Mechanical and } \\
\text { electromechanical } \\
\text { properties }\end{array}$ & Materials & References \\
\hline $\begin{array}{l}\text { AFM-based } \\
\text { indentation (e.g., } \\
\text { ramp, force } \\
\text { relaxation, creep, } \\
\text { dynamic oscillatory } \\
\text { loading) }\end{array}$ & $\begin{array}{l}F_{n} \sim 0.1 \mathrm{nN}-1 \mu \mathrm{N} \\
D \sim 0.1-1 \mu \mathrm{m}\end{array}$ & $\begin{array}{l}\text { Pyramidal and } \\
\text { spherical (colloidal) } \\
\text { probes on planar } \\
\text { surfaces }\end{array}$ & $\begin{array}{l}E_{\text {ind }}, \tau,\left|E^{*}\right|, \delta, E^{\prime}, \\
E^{\prime \prime}\end{array}$ & $\begin{array}{l}\text { Native, treated, and } \\
\text { engineered } \\
\text { cartilage tissue; } \\
\text { single cartilage } \\
\text { cells }\end{array}$ & $\begin{array}{l}11,12 \\
91-105 \\
136,162\end{array}$ \\
\hline $\begin{array}{l}\text { AFM-based high- } \\
\text { resolution force } \\
\text { spectroscopy }\end{array}$ & $\begin{array}{l}F_{n} \sim 0.01-100 \mathrm{nN} \\
D \sim 1 \mathrm{~nm}-1 \mu \mathrm{m}\end{array}$ & $\begin{array}{l}\text { Local region at the } \\
\text { apex of the } \\
\text { pyramidal and } \\
\text { spherical probes on } \\
\text { planar surfaces }\end{array}$ & $F_{a}, E_{a}, \rho_{m}, \sigma_{s}$ & $\begin{array}{l}\text { Cartilage } \\
\text { extracellular matrix } \\
\text { macromolecular } \\
\text { assemblies }\end{array}$ & $\begin{array}{c}79,111,112 \\
144,145 \\
150,163\end{array}$ \\
\hline $\begin{array}{l}\text { AFM-based } \\
\text { single-molecule } \\
\text { force spectroscopy }\end{array}$ & $\begin{array}{l}F \sim 0.01-100 \mathrm{nN} \\
D \sim 1 \mathrm{~nm}-300 \mathrm{~nm}\end{array}$ & $\begin{array}{l}\text { Nonspecific } \\
\text { adsorption or } \\
\text { covalent binding to } \\
\text { pyramidal and } \\
\text { spherical probes }\end{array}$ & $L_{p}, L_{c}$ & $\begin{array}{l}\text { Single cartilage } \\
\text { extracellular matrix } \\
\text { molecules }\end{array}$ & 114,115 \\
\hline $\begin{array}{l}\text { AFM-based lateral } \\
\text { force microscopy }\end{array}$ & $F_{l} \sim 0.01-100 \mathrm{nN}$ & $\begin{array}{l}\text { Pyramidal and } \\
\text { spherical (colloidal) } \\
\text { probes on planar } \\
\text { surfaces }\end{array}$ & $\mu$ & $\begin{array}{l}\text { Native and treated } \\
\text { cartilage tissue; } \\
\text { cartilage } \\
\text { extracellular matrix } \\
\text { macromolecular } \\
\text { assemblies }\end{array}$ & $\begin{array}{r}118,119 \\
120,122 \\
123,164\end{array}$ \\
\hline $\begin{array}{l}\text { Instrumented } \\
\text { (depth-sensing) } \\
\text { indentation }\end{array}$ & $\begin{array}{l}F \sim 1 \mu \mathrm{N}-1 \mathrm{~N} \\
D \sim 1 \mathrm{~nm}-100 \mu \mathrm{m}\end{array}$ & $\begin{array}{l}\text { Berkovich, } \\
\text { spherical, and } \\
\text { conical probes on } \\
\text { planar surfaces }\end{array}$ & $E_{\text {ind }}, \tau$ & $\begin{array}{l}\text { Native, treated, and } \\
\text { engineered } \\
\text { cartilage tissue }\end{array}$ & $\begin{array}{l}86,94 \\
106-109\end{array}$ \\
\hline Optical tweezers & $\begin{array}{l}F \sim 0.1-100 \mathrm{pN} \\
D \sim 1 \mathrm{~nm}-10 \mu \mathrm{m}\end{array}$ & $\begin{array}{l}\text { Two opposing } \\
\text { spheres }\end{array}$ & $L_{p}, L_{c}$ & $\begin{array}{l}\text { Single cartilage } \\
\text { extracellular matrix } \\
\text { molecules }\end{array}$ & $\begin{array}{l}129,130 \\
165,166\end{array}$ \\
\hline $\begin{array}{l}\text { Surface force } \\
\text { apparatus }\end{array}$ & $\begin{array}{l}F_{n}, F_{l} \sim 0.01-10 \\
\mathrm{mN}\end{array}$ & Crossed cylinders & $\mu$ & $\begin{array}{l}\text { Cartilage } \\
\text { extracellular matrix } \\
\text { macromolecular } \\
\text { assemblies }\end{array}$ & $\begin{array}{c}53,124-127 \\
167\end{array}$ \\
\hline
\end{tabular}

\footnotetext{
aAbbreviations: $E_{\text {ind }}$, indentation modulus; $\tau$, force relaxation time constant; $\left|E^{*}\right|$, complex dynamic indentation modulus; $\delta$, phase angle between oscillatory indentation force and depth; $E^{\prime}$, indentation storage modulus; $E^{\prime \prime}$, indentation loss modulus; $F_{a}$, adhesion force; $E_{a}$, adhesion energy; $\rho_{m}$, volume charge density; $\sigma_{s}$, surface charge density; $L_{p}$, polymer persistence length from the worm-like chain model; $L_{c}$, polymer contour length; $\mu$, lateral linearity ratio $\left(=d F_{l} / d F_{n}\right)$.
}

where $v$ is the Poisson's ratio of the specimen and $E_{i}$ and $v_{i}$ are Young's modulus and Poisson's ratio of the indenter, respectively. For indentation of cartilage using indenters made of hard materials (e.g., diamond, silicon nitride, silicon, and borosilicate), $E_{i} \gg E_{\text {ind }}$ and the second term of Equation 2 can typically be neglected.

The Oliver-Pharr method (84) assumes that the unloading portion is elastic and timeindependent. It can account for nonlinearities through use of a contact area that captures the behavior of materials that undergo elastic and permanent deformation upon indentation (84). 
One difference between cartilage indentation and the Oliver-Pharr method is that cartilage undergoes permanent damage only at very high strains [of 30-40\% (85)]. These strains are typically much greater than those applied via nano- or microindentation (86), and hence permanent deformation of cartilage is expected to be negligible in such cases. Instead, cartilage exhibits timeand rate-dependent mechanical behavior and undergoes poroviscoelastic relaxation and creep (30), which dramatically affect both the loading data and the unloading data. Hence, estimations of $E_{\text {ind }}$ using the Oliver-Pharr method (84) may incorporate the effects of elastic resistance to multiaxial compression combined with the rate-dependent effects associated with poroviscoelastic relaxation/creep at a given indentation rate. In addition, at the length scale of indentation, anisotropic properties may be present due to cartilage nano- and microstructural features, such as collagen fibril alignment (87), the presence of chondrocytes, and local differences in aggrecan density. Given the limitations of the Oliver-Pharr method regarding interpretation of cartilage loading, the calculated $E_{\text {ind }}$ is a measure of effective multiaxial indentation stiffness and can be utilized to assess trends in the mechanical response of cartilage to different experimental conditions at a given indentation rate. For example, there are sample-to-sample comparisons and location-specific differences, as discussed in detail in Sections 3.1 and 3.2.

A second method for the calculation of $E_{\text {ind }}$ is based on Hertzian contact theory (88), which assumes material isotropy, homogeneity, and linear elasticity. Lin \& Horkay (2) have comprehensively reviewed indentation on compliant biological tissues and gels. This review discusses and summarizes the analytical formulations on the basis of the elastic Hertzian contact theory, as well as the JKR (Johnson-Kendall-Roberts) (89) and DMT (Derjaguin-Muller-Toporov) (90) modifications of the original Hertzian model that incorporate adhesion-induced deformation and corresponding changes in the contact area. Useful algorithms for determination of the tip-sample contact point for irregular data sets, such as the golden section search, are also presented in Reference 2. Examples of irregular data sets include those with excessive noise, significant adhesion and repulsion, imperfect tip-sample contact, or large strains. In Hertzian models, plasticity and time-dependent poroviscoplasticity are assumed to be negligible, and $E_{\text {ind }}$ is approximated from the loading portion of the force-depth curves. Two common indenter geometries utilized for the indentation of cartilage are spherical (colloidal) and pyramidal (standard silicon nitride) probe tips (91). For spherical probe tips,

$$
F=\frac{4}{3} \frac{E_{\text {ind }}}{\left(1-v^{2}\right)} R^{1 / 2} D^{3 / 2}
$$

where $F$ is the indentation force, $D$ the indentation depth, $R$ the tip radius, and $v$ the Poisson's ratio [e.g., $v=0.1$ for young bovine cartilage (92)]. For pyramidal $(R \ll D)$ probe tips (93),

$$
F=\frac{1.4906}{2} \frac{E_{\text {ind }}}{\left(1-v^{2}\right)} \tan \alpha D^{2}
$$

where $\alpha$ is the half open angle of the pyramidal face.

Other techniques such as indentation force relaxation $(86,94)$ and nanoscale dynamic oscillatory loading (12) have been applied to quantify the time-dependent mechanical behavior of cartilage. For force relaxation, a typical ramp indentation is applied, followed by a hold period during which the change in indentation force $F$ is recorded while the indentation depth $D$ is maintained relatively constant (12). The instantaneous indentation modulus $E_{\text {ind }}(t)$ can then be calculated using the Hertzian analytical models (Equations 3 and 4). The equilibrium indentation modulus (after completion of relaxation), $E_{\text {ind }, 0}$, and the relaxation time constant, $\tau$, can be calculated via a nonlinear least-squares regression based on a spring-dashpot time-dependent model:

$$
E_{\text {ind }}(t)=E_{i n d, 0}+\sum_{i} E_{i n d, i} \exp \left(-t / \tau_{i}\right)
$$


where each pair of $\tau_{i}$ and $E_{i n d, i}$ represents the different modes of relaxation that, for cartilage, may be attributable to visco- and/or poroelasticity (30).

By superimposition of a nanometer-scale sinusoidal deformation during the hold period of a force relaxation experiment at the static indentation depth $D$ (after a time sufficient to allow force relaxation), a dynamic oscillatory loading experiment can probe the nonequilibrium cartilage response by assessing the frequency-dependent complex dynamic modulus $\left|E^{*}\right|$ and the phase angle $\delta$ between the measured oscillatory force and the applied oscillatory indentation. In the limit of infinitesimal sinusoidal deformation $(\tilde{D} \ll D),\left|E^{*}\right|$ can be estimated via a Taylor series expansion of the analytical models, e.g., Equations 3 and 4:

$$
\begin{gathered}
\tilde{F} \approx 2 \frac{\left|E^{*}\right|}{\left(1-v^{2}\right)} R^{1 / 2} D^{1 / 2} \tilde{D}, \\
\tilde{F} \approx 1.4906 \frac{\left|E^{*}\right|}{\left(1-v^{2}\right)} D \tilde{D} \tan \alpha,
\end{gathered}
$$

for spherical and pyramidal tips, respectively. The complex modulus $E^{*}$ can be represented in terms of its real component (the storage modulus, $E^{\prime}=\left|E^{*}\right| \cos \delta$ ) and its imaginary component (the loss modulus, $E^{\prime \prime}=\left|E^{*}\right| \sin \delta$ ). As discussed in more detail in Sections 3.1-3.4, these methods have been widely applied to quantify mechanical properties of native and engineered cartilage $(11,12,86,94,95,106-109,162)$. At the macroscale, energy dissipation in cartilage subjected to compressive loads (as characterized by the loss modulus) has been ascribed to poroelastic behavior with additional contributions from solid matrix viscoelasticity; these loss mechanisms are just beginning to be studied at the nanoscale.

\subsection{Atomic Force Microscopy-Based Force Spectroscopy}

AFM has also been used to quantify molecular-level interactions of cartilage ECM macromolecules in a variety of modes. As shown in Table 2, these include normal HRFS (loading direction perpendicular to the sample plane) (79), LFM (loading direction parallel to the sample plane) (80), and SMFS (tensile stretching of individual molecules) (6). AFM-based force spectroscopy employs cantilever force sensors with spring constants $k \sim 0.01-0.1 \mathrm{~N} \mathrm{~m}^{-1}$, which enables the detection of piconewton-level forces (79) and aims to probe the molecular-level interactions rather than internal solid/fluid mechanical properties of the bulk of the specimen (as carried out in nanoindentation). The absolute value of the tip-sample separation distance can be obtained using microcontact printing sample preparation methods (121) in conjunction with contact mode AFM imaging to measure the compressed layer height and to provide accurate information on the zero-distance position (110). AFM-based force spectroscopy has been applied to measure the molecular-level repulsive interactions of end-attached monolayers of cartilage ECM macromolecules such as CSGAG $(79,111)$ and aggrecan $(110,112)$. The molecular origins of cartilage HRFS data have been quantitatively investigated through comparison with a number of Poisson-Boltzmann-based electrostatic double-layer models, including the surface charge model, the volume charge model, and the charged-rod model (113):

- The surface charge model represents the fixed charges on aggrecan/GAGs as a constant charge density on a surface:

$$
\nabla^{2} \Phi=\frac{2 F C_{0}}{\varepsilon_{W}} \sinh \left(\frac{F \Phi}{R T}\right) .
$$


- The volume charge model represents the region of aggrecan/GAG brushes with uniform fixed charge density $\rho_{\text {volume: }}$

$$
\nabla^{2} \Phi=\frac{2 F C_{0}}{\varepsilon_{W}} \sinh \left(\frac{F \Phi}{R T}\right)-\frac{\rho_{\text {volume }}}{\varepsilon_{W}} .
$$

- The charged-rod model represents each GAG chain as a finite charged rod with a uniform volume charge density $\rho_{\text {rod }}$ :

$$
\nabla^{2} \Phi=\frac{2 F C_{0}}{\varepsilon_{W}} \sinh \left(\frac{F \Phi}{R T}\right)-\frac{\rho_{\text {rod }}}{\varepsilon_{W}} .
$$

In Equations 8-10, $\Phi$ is the electrical potential, $F$ is the Faraday constant $\left(96,500 \mathrm{C} \mathrm{mol}^{-1}\right), R$ is the universal gas constant $\left[8.314 \mathrm{~J}(\mathrm{~mol} \cdot \mathrm{K})^{-1}\right], T$ is the absolute temperature $(298 \mathrm{~K}), \varepsilon_{W}$ is the bulk solution permittivity $\left[6.92 \times 10^{-10} \mathrm{C}\left(\mathrm{N} \cdot \mathrm{m}^{2}\right)^{-1}\right]$, and $C_{0}$ is the bath salt concentration [mol-liter ${ }^{-1}$ ]. Among these three models, the charged-rod model is able to account for the nanoscale heterogeneity in the electrostatic potential and hence results in a better agreement with experimental data on the nanomechanical behavior of GAGs and aggrecan, as discussed in detail in Section 4.1.

When samples are prepared such that the molecules of interest are sparsely distributed on a planar substrate and/or the tip radius is reduced to the length scale of individual molecules, HRFS is used as an SMFS tool to study the tensile extensibility and stiffness of single molecules. For example, the force $(F)$ versus tip-sample separation distance (equivalent to the macromolecular extension, $L$ ) profiles of CS-GAG, KS-GAG, HA (114), and aggrecan (115) molecules have been measured by stretching single molecules in aqueous solution, which can then be fitted to the extensible worm-like chain model (116) to quantify molecular properties such as contour length $L_{c}$ and persistence length $L_{p}$ :

$$
F=\frac{k T}{L_{p}}\left[\frac{1}{4}\left(1-\frac{L}{L_{c}}+\frac{F}{\phi}\right)^{-2}+\frac{L}{L_{c}}-\frac{F}{\phi}-\frac{1}{4}\right],
$$

where $T$ is the absolute temperature, $k$ the Boltzmann constant, and $\phi$ the intrinsic elastic enthalpic stiffness of the polymer chain.

Using AFM-based LFM, researchers can also quantify lateral forces $L$ by scanning the tip horizontally across the sample surface at applied normal forces $F$. The absolute units of the lateral force, and hence the friction coefficient $\mu(=d L / d F)$, can be determined via appropriate calibrations such as the wedge method (117). LFM has since been applied to study microscale cartilage friction in the absence $(118,164)$ and presence $(119)$ of enzymatic treatments and the lubrication effect of cartilage surface molecules $(120,163)$. In conjunction with microcontact printing (121), LFM has also been utilized to quantify the shear properties of aggrecan $(122,123)$ (in this case, $\mu$ is more accurately referred to as the lateral linearity coefficient) by monitoring the compressive strain and lateral forces simultaneously.

\subsection{Surface Force Apparatus}

The SFA measures the vertical and lateral forces between two crossed, transparent mica cylinders $(R \sim 1 \mathrm{~cm})$ in aqueous solutions while monitoring the separation distance between them at $\sim 1-\AA$ resolution using a multiple-beam interference fringe technique $(82,83)$. Established as a robust technique to probe interactions between two layers at small separation distances, SFA has been applied to directly probe the lubrication properties of $\operatorname{HA}(53,124,125)$ and lubricin $(126,127)$ via different adsorption methods and molecular configurations. These studies have shown the potential to yield insights into the underlying lubrication mechanisms of cartilage. 


\subsection{Optical Tweezers}

Optical tweezers operate by focusing a laser to a diffraction-limited spot with a high-numericalaperture microscope objective to trap a dielectric particle (6). An interferometer monitors the displacement of the bead with nanometer-level accuracy. The optical field can then polarize the dielectric particle and result in force directed along the gradient of the optically induced dipoles at $\sim 0.1$-pN resolution (128). As a versatile tool for single-cell and single-molecule manipulation, optical tweezers are another tool for SMFS, which measures the force and extension of single cartilage ECM macromolecules, including HA (129, 166, 167), type II collagen (130), and proteoglycan aggregates $(131,165)$. As in AFM-based SMFS, the extensible worm-like chain model of Equation 11 (116) can be fit to the data and used to estimate $L_{c}$ and $L_{p}$.

\section{NANOMECHANICAL PROPERTIES OF WHOLE TISSUE}

Over the past decade, a number of studies applying nanomechanical techniques have successfully probed cartilage properties associated with microstructural features (91, 99-103, 105), age and disease (96), and tissue repair (106). Additionally, nanoscale methodologies are useful when the volume of material available is too small or irregularly shaped for larger-scale analyses. Examples include differentiation of zonal and regional heterogeneity, detection of early matrix degradation upon aging and osteoarthritis, and evaluation of tissue-engineered matrix properties. These applications are elaborated below.

\subsection{Regional and Zonal Nanomechanical Heterogeneity of Native and Tissue-Engineered Cartilage}

One advantage of nanoindentation is its ability to detect spatial heterogeneities in cartilage mechanical properties, which are thought to exist at a hierarchy of length scales. Ebenstein et al. (106) utilized an instrumented nanoindenter with a spherical probe tip $(R \sim 100 \mu \mathrm{m})$ to test early-stage rabbit cartilage repair tissue up to $\sim 60 \mu \mathrm{N}$ force and $1.3 \mu \mathrm{m}$ indentation depth (Figure $2 a$ ). Indentation stiffness $S$ (in micronewtons per nanometer, as defined by Equation 1) was measured for both native (control) and repair cartilage tissue; native cartilage showed a significantly higher stiffness value (Figure $2 \boldsymbol{b}$ ). Differences in stiffness from the proximal and distal sites (Figure $2 \boldsymbol{b}$ ) were associated with the relative amount of proteoglycan content and the clear formation of chondrocyte cell organizations measured via histology (106). The proximal sites showed indications of ongoing healing of the cartilage with hyaline-like morphology that is similar to that of native articular cartilage; the distal sites were similar to fibrous tissue, with inferior mechanical properties. Using the same technique, Li et al. (107) found zonal differences in stiffness of native rabbit cartilage in distal and proximal sites (Figure $2 c$ ). This difference depended on the structural features of the superficial zone (e.g., thickness, collagen network orientation), with a significant positive correlation between superficial zone thickness and stiffness (107). This technique was also used to evaluate miniature pig hyaline and repair cartilage with a Berkovich tip in phosphatebuffered saline solution (109). The repair cartilage showed lower indentation stiffness compared with native cartilage, consistent with lower proteoglycan content measured via histology. Therefore, the technique of instrumented nanoindentation was suggested to be capable of assessing the temporal evolution of mechanical properties of repair tissue.

Gupta et al. (108) used nanoindentation and the Oliver-Pharr method (84), in conjunction with back-scattering electron imaging, to investigate the correlation between the mineral content and reduced modulus $E_{r}$ in the zone of calcified cartilage (ZCC). The ZCC is the interface 
a

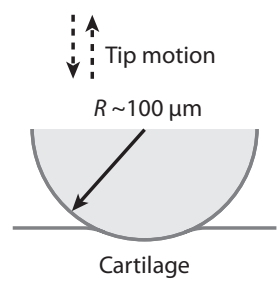

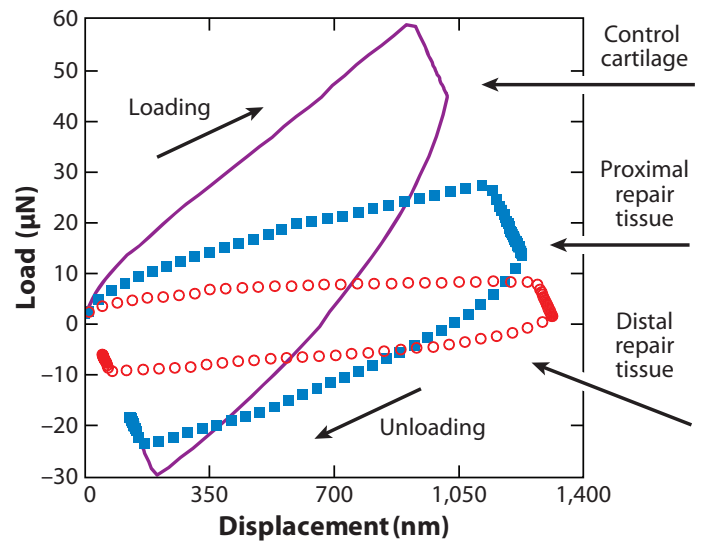
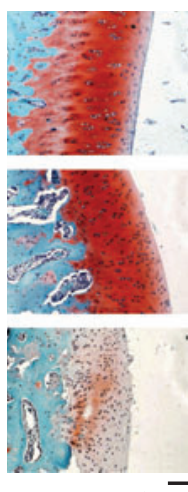

$50 \mu \bar{m}$
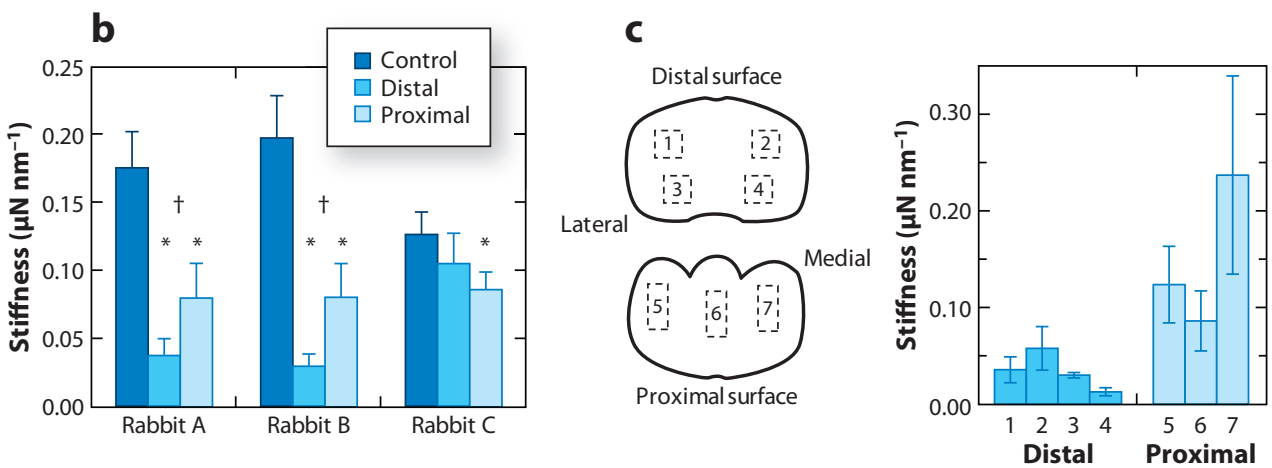

Figure 2

Instrumented microindentation of cartilage tissue. (a) Representative load-displacement curves using a spherical indenter tip $(R \sim 100 \mu \mathrm{m})$ in a hydrated state and safranin-O-stained histological sections (red stains for aggrecan) for control and repair tissue via a rabbit model (rabbit B of panel $b$ ). $(b)$ Indentation stiffness $S$ for three tissue regions (control, distal repair, and proximal repair) of three rabbits (mean $\pm \mathrm{SD}$ ). Asterisks denote that the repair region is significantly different from the control region $(p<0.05)$. Daggers denote that the distal and proximal regions are significantly different from each other $(p<0.05)$. Panels $a$ and $b$ adapted with permission from Reference 106. (c) Schematic of seven different regions of interest (ROI) for a normal rabbit metacarpophalangeal joint tested by microindentation. (Left) Marked boxes indicate a square pattern of four indentations (labeled 1-4) at each distal ROI or three indentations (labeled 5-7) at each proximal ROI (not drawn to scale). Adapted with permission from Reference 107. (Right) The plot shows the corresponding indentation stiffness $S$ for the seven ROI shown at left (mean \pm SD). Data adapted from table 3 in Reference 107.

where fracture occurs between bone and cartilage and abnormal thickening and progression occur upon weight increase or osteoarthritis (132). By indentation of cartilage specimens from human patellae with a Berkovich indenter, significantly lower indentation stiffness was found in the ZCC compared with under the bone for similar mineral content. This difference was suggested to be due to the differences between the organic matrices in these two tissues. A decreasing trend in mineral content and indentation stiffness was also detected from the ZCC out to the unmineralized cartilage at the interface (tidemark) between them. These features at the bone-cartilage junction were hence suggested to preserve mechanical integrity between the two tissues and to reduce the probability of crack formation and propagation (108). 
Jurvelin et al. (98) utilized AFM-based indentation with a pyramidal probe tip and $\sim 100-\mathrm{nm}$ indentation depth to distinguish between native and proteoglycan-depleted cartilage disks. Subsequently, Mao and colleagues (99-103) employed this method to quantify age-related regional, zonal (depth-dependent), and territorial heterogeneities (see Figure $\mathbf{1} \boldsymbol{b}$ for the last two cases) in cartilage mechanical properties using a rabbit model. In this case, $E_{\text {ind }}$ was estimated using the Hertz model (88) and differed significantly for different regions on the adult articular surface (99), but not for the neonatal surface (101). On the basis of its spatial homogeneity, neonatal cartilage was suggested to be a dynamic matrix that is still undergoing development and maturation. In addition, $E_{\text {ind }}$ increased from the outermost superficial zone to the innermost calcified zone for both articular (102) and growth plate (99) cartilage. The observed regional and zonal variations in mechanical properties were attributed to the differences in structure, composition, and chemical properties of the matrix (100).

Heterogeneity was also observed within each of the cartilage cellular zones. In vivo, each chondrocyte is surrounded by a $2-3-\mu$ m-thick pericellular matrix (PCM), which is interconnected to the further removed territorial and interterritorial matrix zones (Figure $1 \boldsymbol{b}$ ) of ECM (Figure 3a) (133). Darling et al. utilized AFM-based nanoindentation with a spherical tip $(R \sim$ $2.5 \mu \mathrm{m}$ ) and the Hertz model (Equation 3) to obtain indentation modulus maps in the PCM and territorial/interterritorial matrices of human, porcine (Figure $3 \boldsymbol{b}$ ), and murine articular cartilage (105). The PCM zone was more compliant than the ECM, with a ratio of $E_{\text {ind,PCM }} / E_{\text {ind,ECM }} \sim 0.35$ for all tested species. A similar trend was observed for rat cartilage, with significantly higher $E_{\text {ind }}$ in the territorial/interterritorial zone than in the pericellular zone. This difference was hypothesized to correlate with different biological functions of these two zones, which are of vital importance to cartilage health (103). Although territorial and interterritorial zones of ECM are responsible mainly for external load support and energy dissipation (18), the more compliant PCM results in significant strain amplification in the vicinity of chondrocytes and functions as a transducer of mechanical signals from the ECM to the chondrocytes (134).

At even smaller length scales, heterogeneity that was associated with macromolecular composition of the ECM was detected. Using a pyramidal (nanosized) tip and the Oliver-Pharr method (84), Stolz and colleagues $(91,97)$ quantified $E_{\text {ind }}$ of porcine articular cartilage, in which a bimodal distribution of $E_{\text {ind }}$ was suggested to correspond to collagen for higher values of $E_{\text {ind }}$ and to aggrecan for lower values of $E_{\text {ind }}$.

\subsection{Maturation- and Pathology-Related Indentation Behavior}

Murine models of degenerative joint disease have been widely utilized during the past decade because of the ability to modify specific tissue development and functional properties using gene knockout and related gene-altering technologies (135). However, the mechanical properties of mouse cartilage are difficult to quantify via conventional macroscopic testing due to the small volume and irregular shape of the tissue. As a result, it has not been possible to draw conclusive statements of how these gene-altering techniques affect cartilage mechanical properties. Stolz et al. (96) employed AFM-based nanoindentation on mouse cartilage using both spherical and pyramidal probe tips to assess the difference in microscale mechanical properties (indentation stiffness $S$ and modulus $E_{\text {ind }}$ ) from the Oliver-Pharr method (84). Effects of both the maturation process and osteoarthritis-like degradation of type IX collagen in the gene knockout were investigated. For normal mouse joints, AFM-based indentation with a pyramidal tip detected a significant increase in stiffness from 1- to 19-month-old mouse joints (Figure 3c). The increase in stiffness was attributed to the observed thickening of collagen fibrils due to the deposition of small proteoglycan molecules onto the collagen fibrils and/or fibril bundling as a consequence of the reduced interfibrillar GAG content. This mechanical property change was ascribed to the change in the nanostructure and 

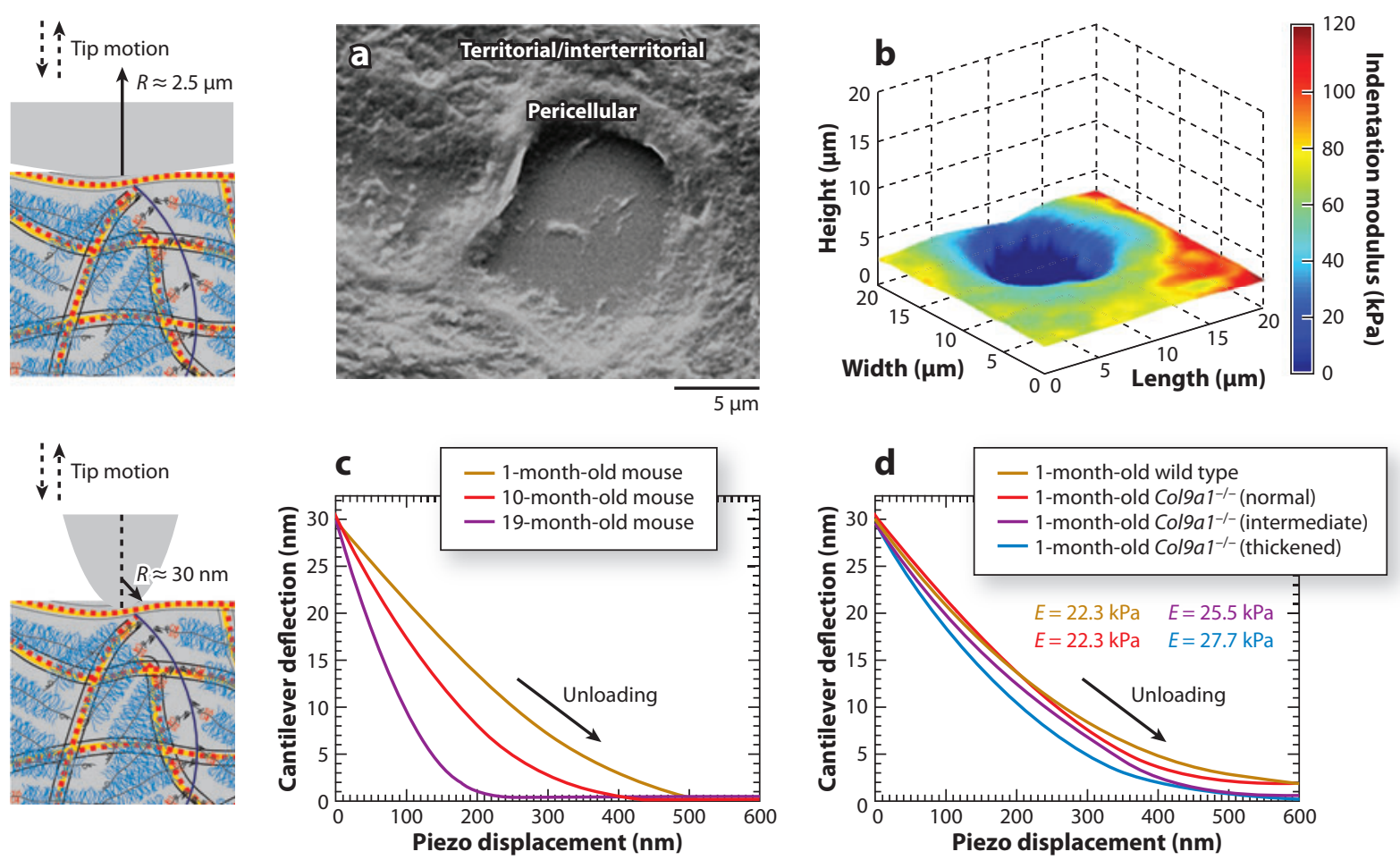

Figure 3

Atomic force microscopy-based nanoindentation on articular cartilage. (Left panels) Schematic of microsized (top) and nanosized (bottom) indenter tips versus cartilage collagen and aggrecan molecular size (drawn to scale). (a) Scanning electron microscope image of pericellular and territorial/interterritorial matrices of middle/deep zone extracellular matrix of porcine articular cartilage, where the hole in the middle represents the region originally occupied by a chondrocyte. $(b)$ Representative height and indentation modulus $E_{\text {ind }}$ spatial map of porcine cartilage using a microspherical tip $(R=2.5 \mu \mathrm{m})$ in phosphate-buffered saline (PBS). Panels $a$ and $b$ adapted with permission from Reference 105. $(c, d)$ Typical nanoindentation force-versus-z-piezo displacement unloading curves on normal and type IX collagen gene-knockout $\left(\right.$ Col9a $\left.1^{-/-}\right)$murine joints via a nanosized pyramidal tip in PBS: $(c)$ aging normal C57BL/6 mice $(n=$ 15 per group) and $(d) 1$-month-old wild-type controls and Col9a $1^{-/-}$mice using a nanosized pyramidal tip $(n=7)$. Panels $c$ and $d$ adapted with permission from Reference 96.

nanomechanical properties of cartilage ECM constituent changes during maturation, e.g., a betterorganized collagen fibrillar network and an increased amount of proteoglycan (96).

Osteoarthritis-like deterioration of cartilage at different disease stages was studied by AFMbased indentation using a mouse model deficient in type IX collagen (96). At early stages of osteoarthritis (demonstrated by the 1-month-old type IX collagen-deficient mouse), histology did not show any obvious microstructural changes compared with normal joints. Nanoindentation using a nanosized tip was able to detect a significantly lower indentation stiffness for the osteoarthritis joints (Figure 3d), associated with nanoscale fibril thickening via AFM imaging. The absence of type IX collagen was thought to alter the molecular architecture and to result in the aggregation of individual collagen fibrils to form thicker fibril bundles. AFM may therefore have the potential to monitor the early onset of osteoarthritis before techniques such as histology and molecular markers can detect changes. In a separate study, mouse joints at different stages of early maturation were investigated using AFM-based indentation with a spherical probe tip (136). The investigators detected differences in cartilage $E_{\text {ind }}$ as well as changes in $E_{\text {ind }}$ caused by inflammation from injection of transforming growth factor- $\beta 1$. 


\subsection{Nanoindentation of the Tissue-Engineered Cell-Associated Matrix}

AFM-based indentation has also been demonstrated as a powerful tool for the evaluation of cartilage engineered neotissue associated with individual cells. $\mathrm{Ng}$ et al. (11) utilized this technique to measure the temporal evolution of tissue-engineered matrix synthesized by single chondrocytes. Here, the mechanical properties of the newly synthesized matrix associated with individual cells were quantified while changes in the matrix composition of GAG and collagen were simultaneously monitored using biochemical methods via toluidine blue and aniline staining assays, respectively (11). To immobilize and prevent dedifferentiation, individual chondrocytes and their associated matrix were confined in a microfabricated pyramidal silicon well during indentation (Figure $4 a$ ). The indentation force-depth curves (using both spherical and pyramidal probe tips) were fit to finite element and analytical models to account for the indentation geometries; both models yielded similar $E_{\text {ind }}$. Although the matrix appeared to accumulate quickly during the early culture period (its thickness remained constant during the tested period), $E_{\text {ind }}$ of the matrix significantly increased with culture duration (Figure 4a). This increase was consistent with the measured
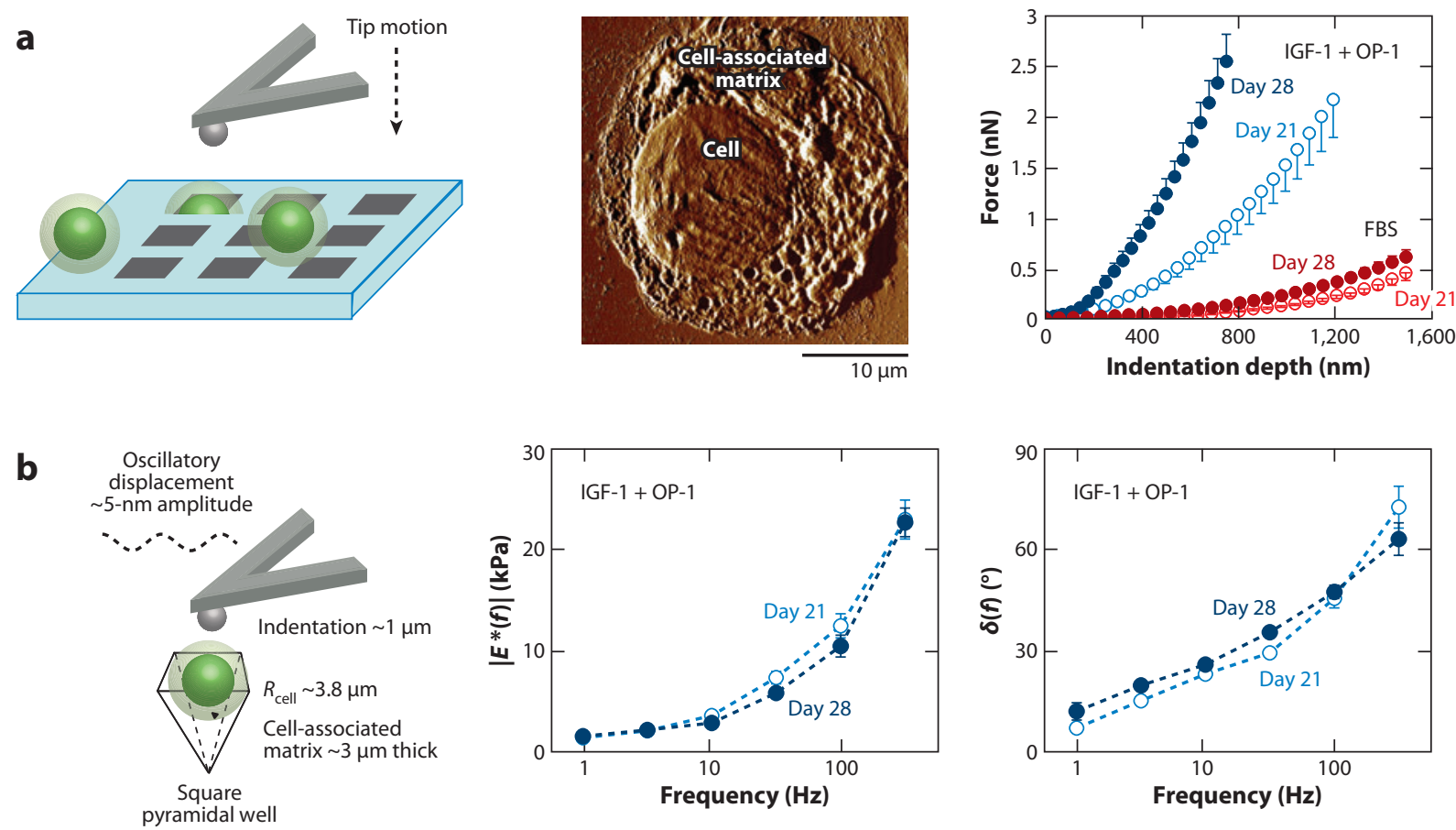

Figure 4

Temporal development of tissue-engineered, chondrocyte-associated matrix. (a) ( $L e f t)$ Schematic of indentation (using a spherical tip $R \sim 2.5 \mu \mathrm{m}$ ) on individual chondrocytes and their cell-associated matrix, which are fixed within pyramidal wells of a silicon microfabricated substrate. (Middle) Tapping-mode atomic force microscopy image of a chondrocyte and newly synthesized tissueengineered cell-associated matrix after 11-day culture in $10 \%$ fetal bovine serum (FBS). (Right) Nanoindentation curve (mean \pm SEM of five loading cycles per cell for $n \geq 5$ cells) on loading of individual chondrocytes with engineered cell-associated matrix (after release from alginate at different times) in culture with 10\% FBS and with insulin-like growth factor-1 (IGF-1) and osteogenic protein-1 (OP-1). Panel $a$ adapted with permission from Reference 11. (b) Dynamic mechanical properties of chondrocytes and their engineered cell-associated matrix (mean $\pm \mathrm{SEM} ; n=5$ cells) using a spherical tip in culture with IGF-1 and OP-1. (Left) Schematic of the dynamic oscillatory nanoindentation. (Middle) Dynamic indentation modulus, $\left|E^{*}\right|$, and (Right) the phase angle, $\delta$, as a function of oscillation frequency after 21 and 28 days of culture. Panel $b$ adapted with permission from Reference 12. 
increase in collagen and GAG and with high-resolution images of the matrix via tapping-mode AFM imaging. The presence of growth factors such as insulin-like growth factor-1 (IGF-1) and osteogenic protein-1 (OP-1) increased the indentation stiffness as well, although there was no significant increase in the amount of GAG and collagen due to addition of these growth factors. This difference in biomechanical versus biochemical results may be due to the effect of these growth factors on molecular organization such as interactions between small leucine-rich proteins that regulate collagen fibrillogenesis, as well as collagen cross-linking (137-139).

Applying an $\sim 5$-nm amplitude dynamic oscillation during nanoindentation to an $\sim 1-\mu \mathrm{m}$ static indentation depth, Lee et al. (12) measured the frequency-dependent poroelasticity of newly synthesized matrix in the frequency range of $1-316 \mathrm{~Hz}$. A monotonic increase in dynamic complex modulus $\left|E^{*}\right|$ and phase angle with frequency suggested the presence of poroviscoelastic rate processes in the cell-associated matrix. Furthermore, the observed increase in modulus and decrease in energy dissipation with longer culture duration were consistent with increased biomacromolecular deposition and altered matrix structure and organization. The presence of IGF-1 and OP-1 growth factors also resulted in an increase in $\left|E^{*}\right|$ and a decrease in $\delta$, suggesting a more elastic response, likely due to compositional and structural changes, including the accumulation of energy-conserving aggrecan proteoglycans (Figure 4b) (140-142).

\subsection{Time-Dependent Indentation Behavior}

Recently, the time-dependent properties of cartilage subjected to small-scale deformations were investigated using instrumented nanoindentation and finite element analysis. With a spherical tip indenter $(R \sim 100 \mu \mathrm{m})$, Gupta et al. (94) performed force relaxation measurements on porcine costal cartilage up to an $\sim 3$-min hold in phosphate-buffered saline (PBS) [pH $\sim 7.4$, ionic strength (IS) $\sim 0.15 \mathrm{M}$ ] that simulated physiological conditions. A fiber-reinforced, poroviscoelastic finite element model was used to determine the material properties. In the model, the proteoglycan content was treated as isotropic, linear, two-dimensional pore pressure elements with hydraulic permeability $k$, and the collagen network was treated as oriented fibers with Young's modulus $E_{\mathrm{Y}}$, Poisson's ratio $v_{m}$, and volume fraction $f_{f}$. The four parameters quantitatively described the nonlinear time-dependent relaxation behavior of the tested cartilage at $\sim 2-3-\mu \mathrm{m}$ indentation depth. As intrinsic viscoelasticity was thought to contribute little to the observed time dependency, lower $k$ values $\left[\sim 10^{-16} \mathrm{~m}^{4}(\mathrm{~N} \cdot \mathrm{s})^{-1}\right]$ compared with other types of cartilage were attributed to the higher proteoglycan content and collagen fiber content of costal cartilage. In a similar study, Miller \& Morgan (86) performed force relaxation on bovine articular cartilage using an $R \sim 50 \mu \mathrm{m}$ spherical tip indenter and compared their results with those from a macroscopic confined-compression test on the same sample. Continuum-level poroelastic finite element models (also referred to as biphasic models) that assumed homogeneous properties of elastic modulus $E$, hydraulic permeability $k$, and Poisson's ratio $v$ were used to predict both elastic and poroelastic time-dependent behavior. Using AFM-based dynamic oscillatory nanoindentation, Han et al. (162) deconvoluted the cartilage visco- and poroelasticity into time and frequency domains, whereby the poroelastic behavior suggested $k$ values similar to those from a macroscale unconfined compression test (24). These studies have demonstrated that nanoindentation has potential applications in probing the time-dependent mechanical characteristics of small, heterogeneous materials such as cartilage and in determining structural features at constituent length scales.

\subsection{Microscale Frictional Properties}

Using a polystyrene spherical probe tip $(R \sim 5 \mu \mathrm{m})$ on normal bovine cartilage surfaces, Park et al. (118) measured via LFM the microscale frictional coefficient $\mu$ (the slope of lateral-versus-applied 
a

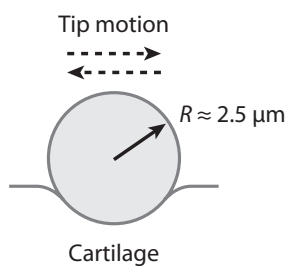

b

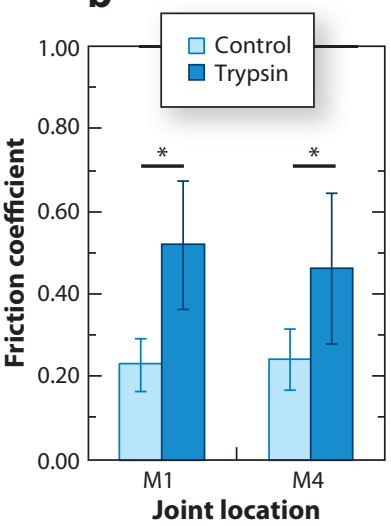

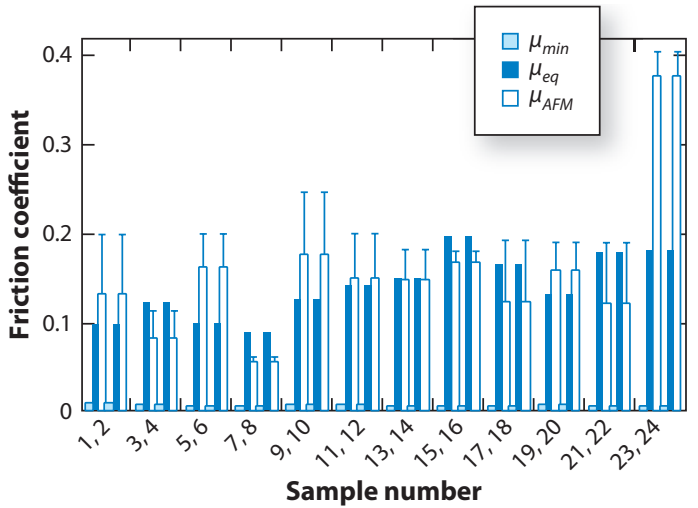

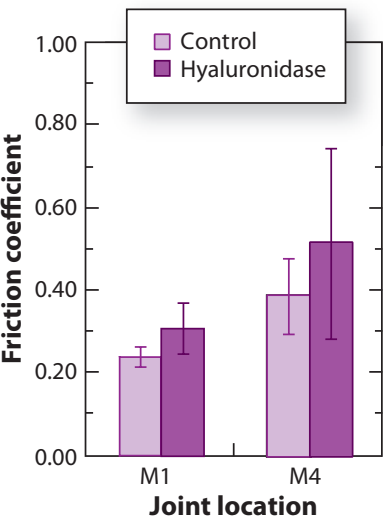

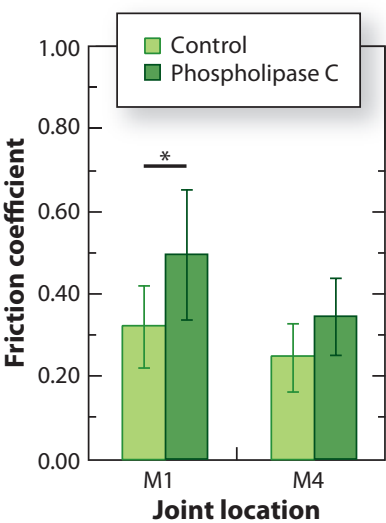

Figure 5

Microfriction of cartilage tissue using lateral force microscopy. (a) Friction coefficients of normal bovine humeral articular cartilage surface from a microscale atomic force microscopy (AFM) test $\left(\mu_{A F M}\right.$; mean \pm SD for three locations on each sample) using a polystyrene spherical tip $(R \sim 2.5 \mu \mathrm{m})$ and macroscopic experiments $\left(\mu_{\min }\right.$ and $\mu_{\text {eq }}$ ) for each specimen pair in phosphate-buffered saline (PBS). Panel $a$ adapted with permission from Reference 118. (b) Effect of enzymatic treatment on friction coefficients $\mu$ of bovine articular cartilage surface regions subject to relatively high (M1) and low (M4) contact pressure in vivo (mean $\pm 95 \%$ confidence interval), measured via a $\mathrm{Si}_{3} \mathrm{~N}_{4}$ pyramidal AFM tip in PBS. Asterisks indicate a significant effect on $\mu$ upon enzymatic treatment; $p \leq 0.0145$ from one-way nested analysis of variance (ANOVA). Panel $b$ adapted with permission from Reference 119.

normal force), yielding a value of $\sim 0.15$, which was consistent with the equilibrium friction coefficient measured by macroscopic tests (Figure $5 \boldsymbol{a}$ ). The relatively high value of $\mu$ compared with the cartilage friction coefficient measured in vivo $(\sim 0.0005-0.04)(21)$ was attributed to the absence of interstitial fluid pressurization and/or critical molecular components in synovial fluid and cartilage. Using the same method with a pyramidal probe tip, Chan et al. (119) assessed microscale friction upon the removal of certain molecular components in both load-bearing locations and non-loadbearing locations on the cartilage surface. Removal of HA or surface-active phospholipids resulted in no significant impact on $\mu$, suggesting that HA and phospholipids do not provide boundary lubrication on the cartilage surface in the absence of other surface-interacting molecules. This observation did not exclude the lubrication and wear protection function of HA in synovial fluid, as was suggested by Tadmor et al. (53). In contrast, trypsin treatment to remove all proteoglycan 
and protein content increased $\mu$ by more than $100 \%$ (Figure $\mathbf{5} \boldsymbol{b}$ ). The effect on friction of trypsin depletion was thought to be due to the degradation of lubricating surface zone proteins as well as of the proteins (albumin, fibronectin) and proteoglycan (aggrecan) to which these superficial zone proteins (lubricin) bind. Coles et al. (143) tested the surface lubrication ability of lubricin by measuring friction upon deletion of the lubricin gene. Knockout of lubricin had limited effect on $\mu$, suggesting that lubricin may not provide significant boundary lubrication on the cartilage surface. In contrast, lubricin may be an effective lubricant when present in synovial fluid (143). In separate studies, molecular mechanisms of surface lubrication by HA and lubricin were directly probed in their molecular assemblies in vitro and are discussed in detail in Sections 4.2 and 4.3. Therefore, microscale frictional studies on cartilage surfaces have yet to provide the final answers to the origins of excellent cartilage biolubrication in vivo.

\section{NANOMECHANICS OF MATRIX MACROMOLECULAR ASSEMBLIES}

To provide fundamental knowledge on cartilage properties from a molecular perspective, it is necessary to study the nanomechanical properties of individual ECM constituents. AFM-based HRFS and LFM have been used to quantify the compression $(79,110-112,144)$, shear $(122,123)$, and self-adhesion (145) of aggrecan in molecular assemblies having similar aggrecan density as those in vivo and tested in aqueous solutions of near physiological concentrations $(0.1 \mathrm{M}, \mathrm{pH} \sim$ 5.6). SFA and LFM have been utilized to study the lubrication properties of HA $(53,120,124$, $125)$ and lubricin $(120,126,127)$.

\subsection{Aggrecan and Its Glycosaminoglycan Side Chains}

Aggrecan is one of the first molecular components to be degraded and released from cartilage in osteoarthritis and is associated with increased activity of aggrecanase enzymes within cartilage tissue (48). Enzymatic proteolysis of aggrecan degrades cartilage biomechanical function, and the tissue is damaged further by continued joint loading. To understand the molecular properties of aggrecan, Seog et al. $(79,111,144)$ measured interactions between CS-GAG covalently grafted on a planar gold substrate and a gold-coated probe tip having $R \sim 50 \mathrm{~nm}$ as a function of bath IS $(0.0001-$ $1.0 \mathrm{M})$ via HRFS. The strong dependence of the measured GAG-GAG interaction force on IS (Figure $6 \boldsymbol{a}$ ) and $\mathrm{pH}$ suggested an important contribution of electrostatic repulsion to GAG-GAG interactions (111). To account for the nanoscale spatial heterogeneity in electrostatic potential, Dean et al. (113) developed a Poisson-Boltzmann-based molecular model that regards CS-GAG as individual cylindrical charged rods of uniform charge density and finite length. An effective compressive modulus associated with electrostatic interactions for the GAG density corresponding to an inter-GAG spacing $s \sim 6 \mathrm{~nm}$ was calculated to be up to $E_{\mathrm{GAG}} \sim 10 \mathrm{kPa}$ at a compressive strain $\varepsilon=0.6$ (Figure $\mathbf{6} \boldsymbol{b}$ ). Steric forces had a minimal contribution to the measured GAG-GAG interactions. $E_{\mathrm{GAG}}$ was approximately two orders of magnitude lower than the cartilage tissue modulus of $\sim 1 \mathrm{MPa}$ at similar tissue strain, due likely to the fact that the GAG density on the end-grafted layer was approximately four times lower than that in cartilage tissue (111). Aggrecan (and hence GAG) is in a prestrained state in uncompressed cartilage, and a $60 \%$ tissue strain would correspond to a much higher molecular strain on the GAG chains (111). However, qualitatively, the deformation of opposing CS-GAGs may mimic certain aspects of intra-tissue loading in which the substrate and tip act as constraints in two dimensions, similar to the in vivo conditions provided by the aggrecan core protein and other ECM molecules in three dimensions.

A closer mimicking of in vivo cartilage molecular interactions was achieved by chemically endgrafting aggrecan macromolecules onto both a planar gold substrate and a microsized spherical 

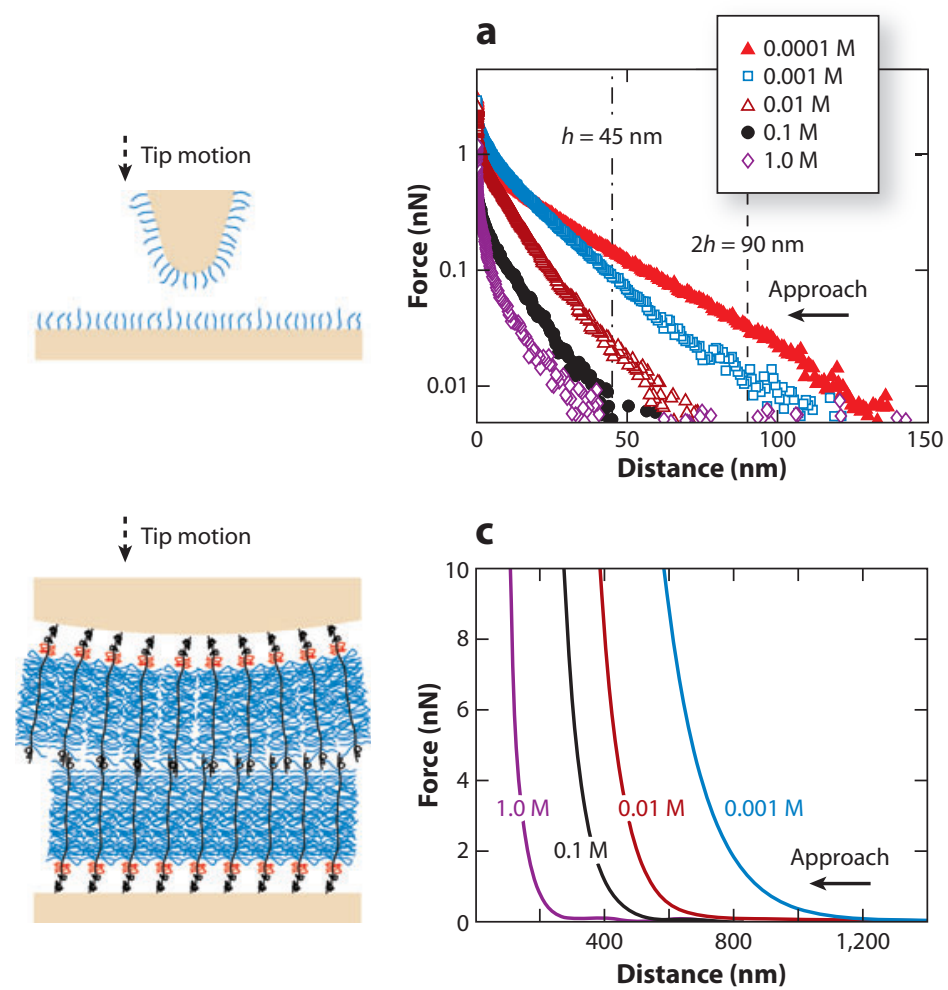
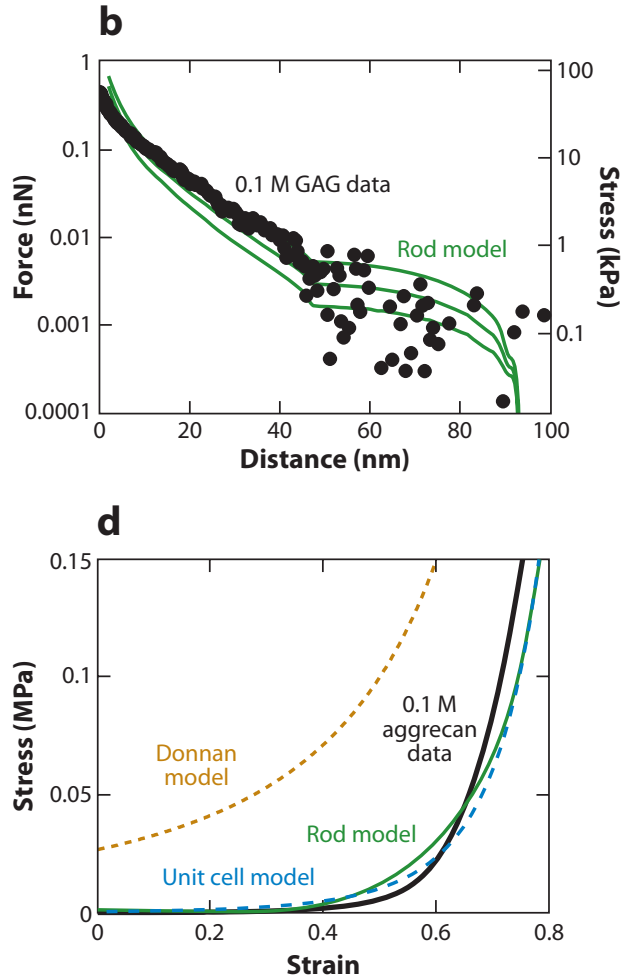

Figure 6

Compressive nanomechanics of cartilage chondroitin sulfate glycosaminoglycans (CS-GAGs) and aggrecan. (Left) Schematic of high-resolution force spectroscopy experiments involving compression between two opposing CS-GAG layers and aggrecan layers. (a) Normal force-versus-separation distance for a nanosized GAG-functionalized probe tip $(R \sim 50 \mathrm{~nm})$ versus a GAG-functionalized planar substrate with GAG-GAG separation distances of $\sim 6.5 \mathrm{~nm}$ in different bath ionic strengths (IS) (0.0001-1.0 M NaCl, $\mathrm{pH} \sim$ 5.6). (b) Comparison of GAG-GAG repulsion high-resolution force spectroscopy data in panel $a$ at $0.1 \mathrm{M}$ IS with the predictions of the interdigitated charged-rod model with parameter values fixed at $[\mathrm{NaCl}]=0.1 \mathrm{M}$, tip radius $R \sim 50 \mathrm{~nm}$, rod height $b=45 \mathrm{~nm}$, radius $w=2 \mathrm{~nm}$, inter-rod distance $s=6-7 \mathrm{~nm}$, and total charge per $\operatorname{rod} Q_{\text {rod }}=-8 \times 10^{-18} \mathrm{C}$. Panels $a$ and $b$ reproduced with permission from Reference 111. (c) Normal force-versus-separation distance between an aggrecan-functionalized spherical tip $(R \sim 2.5 \mu \mathrm{m})$ and an aggrecan-functionalized planar gold substrate in different bath IS (0.001-1.0 M NaCl, pH 5.6). (d) Comparison of converted stress-strain aggrecan-aggrecan repulsion data in panel $c$ at $0.1 \mathrm{M}$ IS with the charged-rod model $(112,113)$, unit cell model $(71,112)$, and volume charge Donnan model (112). Panels $c$ and $d$ adapted with permission from Reference 112.

tip $(R \sim 2.5 \mu \mathrm{m})$ at a physiologically realistic packing density (Figure $6 c)(110,112)$. Microcontact printing (121) was employed to fabricate a micropatterned sample of aggrecan and a neutral self-assembled monolayer with well-defined boundaries (110). This self-assembled monolayer, with negligible height compared with that of aggrecan, functioned as a reference plane, and hence the compressive interactions between two opposing aggrecan layers consisting of $\sim 10^{3}$ aggrecan molecules could be quantified via AFM (Figure $6 c$ ) $(110,112)$. Similar to the GAGGAG interactions, due to the presence of electrostatic repulsion, marked IS dependency and highly nonlinear compressive resistance were observed for the aggrecan system (Figure $6 \boldsymbol{c}$ ). The measured GAG-GAG interactions at physiologically relevant IS $(0.1 \mathrm{M})$ were compared with the predictions by the Poisson-Boltzmann-based theoretical models (Figure $6 \boldsymbol{d}$ ). The homogeneous macroscopic volume charge model (Equation 9) overestimated the measured aggrecan interactions 
for all strains of interest. Both a two-dimensional unit cell model [linear polyelectrolyte surrounded by aqueous electrolyte (71)] and a three-dimensional charged-rod model [nonzero fixed charge density within the cylindrical rods (GAGs)] (Equation 10) (113) accounted for the molecularlevel spatial heterogeneity at fixed charge density and intermolecular electrostatic potential and hence resulted in much better agreement with experimental data than did the volume charge model (Figure $6 \boldsymbol{d}$ ). Using a coarse-grained molecular model, Bathe et al. (72, 73) predicted the IS and $\mathrm{pH}$ dependency of CS-GAG conformations and the resulting osmotic pressure in aqueous solutions on the basis of the free-energy contribution from bonded and nonbonded (electrostatic, steric) energy as well as the translational entropy. This simulation also yielded results similar to those of nanomechanical experiments (112); both studies suggested that molecularlevel electrostatic repulsion contributes significantly to the compressive stiffness of aggrecan. In a separate study modeling the interactions between two aggrecan molecules, Nap \& Szleifer (74) drew similar conclusions on the effect of $\mathrm{pH}$ and IS by using a more detailed molecular model that further included the GAGs' conformational entropy. This model also suggested a low degree of interdigitation between two aggrecan molecules under physiologically relevant conditions, which may explain the lubrication properties of the polyelectrolyte layers in cartilage (74).

Shear properties were assessed using a model system of two opposing end-grafted aggrecan layers in aqueous solutions via $\operatorname{LFM}(123,145)$. The measured lateral force depended linearly on the applied normal force (Figure 7a). The slope of the lateral versus normal force was denoted as the lateral linearity ratio $\mu$. The increase in $\mu$ with increasing IS $(0.001-1.0 \mathrm{M})$ was due to the larger aggrecan compressive strain that resulted from decreased electrostatic interactions for the same normal force. At the same aggrecan compressive strain, the lateral force was higher at lower IS, given the presence of stronger electrostatic repulsion. A significant rate dependence of $\mu$ (Figure $7 \boldsymbol{b}$ ) suggested the presence of viscoelasticity and fluid flow-related poroelasticity, and this rate dependence was also more pronounced at higher IS, as the nonelectrostatic components became more important. The addition of divalent $\mathrm{Ca}^{2+}$ ions-which may be physiologically relevant (18) - led to a decrease in the shear and compressive resistance of the aggrecan layer. This is due to the fact that the divalent $\mathrm{Ca}^{2+}$ ions preferentially distribute closer to GAGs than do monovalent ions (146), and divalent $\mathrm{Ca}^{2+}$ ions can also form ion bridges (147) between the GAG chains to decrease the net repulsion $(148,149)$.

Despite negligible adhesion measured upon detaching of the two opposing aggrecan layers immediately after compression (112), self-adhesion was observed after a sufficient dwell (equilibration hold) time following compressive loads $\sim 50 \mathrm{nN}$ at $0.001-0.1 \mathrm{M}$ IS (Figure $7 c$ ). This aggrecan self-adhesion is expected to result from a fairly complex balance between a host of possible attractive and repulsive (mostly electrostatic) interactions, similar to most biomacromolecular systems. Physical entanglements, hydrogen bonding, hydrophobicity, and van der Waals interactions are expected to contribute to the measured self-adhesion. Aggrecan self-adhesion increased with increasing surface equilibration time and IS (i.e., a decrease in intermolecular repulsion) (Figure $7 d$ ). In addition, the presence of $\mathrm{Ca}^{2+}$ at in vivo concentrations of $\sim 2-4 \mathrm{mM}$ (18) may result in the ion-bridging effects associated with multivalent ions (147) and may significantly enhance aggrecan self-adhesion. This observed self-adhesion, as measured in physiological-like conditions, is expected to be present in vivo and may be an important factor contributing to the self-assembled architecture and integrity of cartilage PCM and ECM in tissue (145).

In addition to studies of aggrecan harvested from native cartilage tissue, Lee et al. (150) investigated the molecular-level structural and mechanical properties of aggrecan synthesized by cells in tissue-engineered constructs. Through the use of a combination of microcontact printing (121) and HRFS (79) techniques, nanostructures and compressive nanomechanical properties of aggrecan macromolecules extracted from native adult equine articular cartilage were compared 

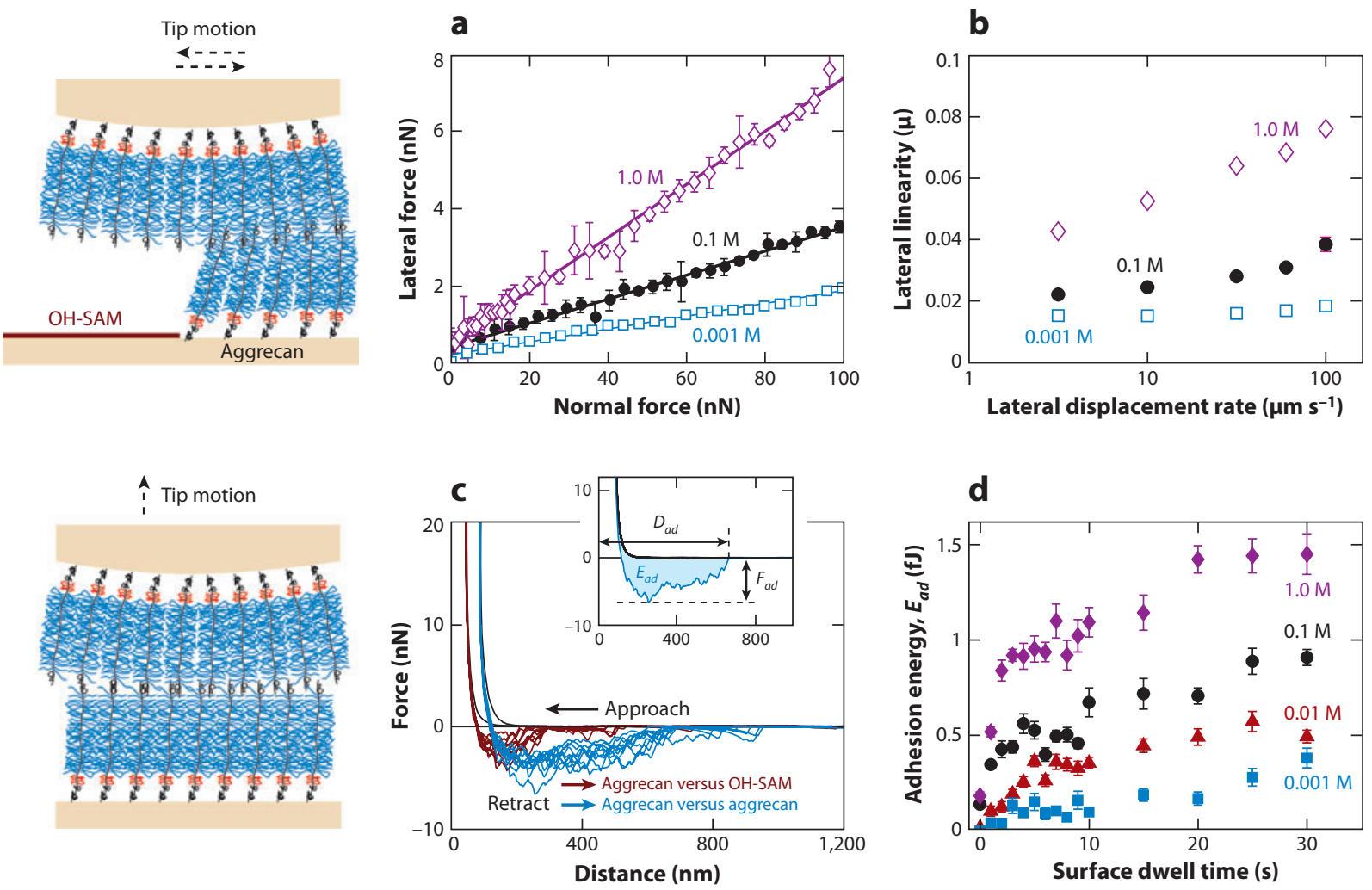

Figure 7

Shear and self-adhesion nanomechanics of cartilage aggrecan. (Left) Schematics of lateral force microscopy experiments involving shear of two opposing aggrecan layers and high-resolution force spectroscopy experiments that probe aggrecan self-adhesion. (a) Lateralversus-applied normal force (mean $\pm \mathrm{SD}, n=8$ different locations) between an aggrecan-functionalized spherical tip $(R \sim 2.5 \mu \mathrm{m})$ and an aggrecan-functionalized planar gold substrate in different bath ionic strengths (IS) (0.001-1.0 M NaCl, $\mathrm{pH} \sim 5.6)$, with corresponding least squares linear regression fit for each IS $\left(R^{2}>0.92\right.$ for all the fits; $95 \%$ confidence interval width of $\left.\mu<0.01\right)$. (b) Lateral proportionality ratio $\mu$ versus lateral tip displacement rate (mean $\pm 95 \%$ confidence interval at $n=8$ different locations; $R^{2}>0.88$ for all data) between the aggrecan tip and aggrecan substrate. Panels $a$ and $b$ adapted with permission from Reference 123. (c) Comparison of force-distance curves obtained via neutral hydroxyl-terminated self-assembled monolayer (OH-SAM) and aggrecan-functionalized tips $(R \sim 2.5 \mu \mathrm{m}$ ) on aggrecan end-grafted planar substrates (IS $=1.0 \mathrm{M}, \mathrm{NaCl}$ aqueous solution, surface dwell time $t=30 \mathrm{~s}$, maximum compressive force $F_{\max } \sim 45 \mu \mathrm{N}$, $z$-piezo displacement rate $z=4 \mu \mathrm{m} \mathrm{s}^{-1}$ ). Different experiments were carried out at 10 different locations, as shown for each probe tip. (Inset) Definition of adhesive interaction distance $D_{a d}$, maximum adhesion force $F_{a d}$, and adhesion energy $E_{a d}$ for each pair of approach-retract force-distance curves. (d) Adhesion energy, $E_{a d}$, versus surface dwell time $t$ between two opposing aggrecan end-grafted layers in $0.001-1.0 \mathrm{M} \mathrm{NaCl}, \mathrm{pH} \sim 5.6\left(F_{\max } \approx 45 \mathrm{~nm}, z \approx 4 \mu \mathrm{m} \mathrm{s}{ }^{-1}\right.$, mean \pm SEM, $n \geq 30$ for each $t$ at each IS). $E_{a d}$ depended significantly on $t$ and IS [two-way analysis of variance (ANOVA), $p_{t}<$ $\left.0.0001, p_{\text {IS }}<0.0001\right]$. Panels $c$ and $d$ adapted with permission from Reference 145 .

with those of aggrecan made by adult equine bone marrow stromal cells (BMSCs) after these cells were stimulated to become cartilage like (chondrogenic) in a self-assembling peptide hydrogel scaffold after 21 days of culture. These two populations of aggrecan exhibited marked differences. The BMSC-aggrecan population had a larger proportion of full-length aggrecan than the native cartilage-aggrecan population, whereas the core protein length $(\sim 400 \mathrm{~nm})$ was similar for the full-length molecules between the populations (Figure 8a). In addition, the GAG chains of 


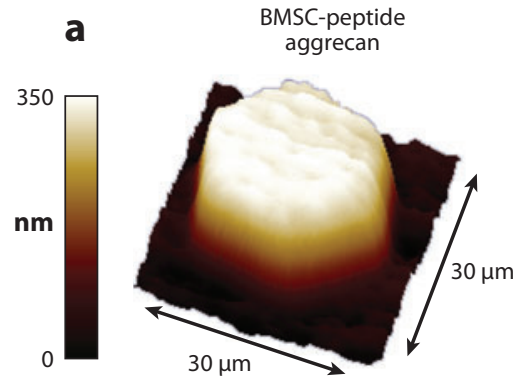

b

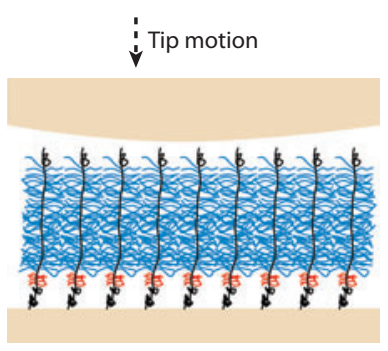

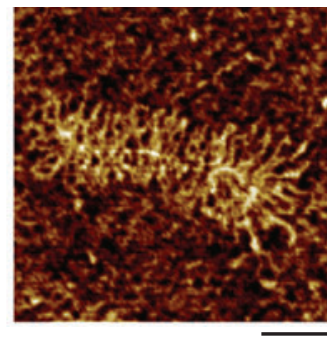

$\overline{100 \mathrm{~nm}}$
Cartilage-extracted aggrecan
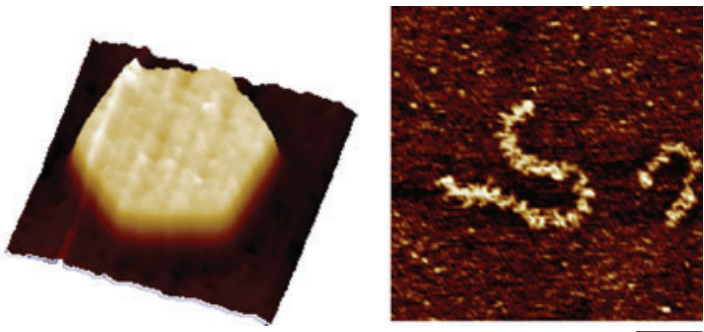

$\overline{100 \mathrm{~nm}}$
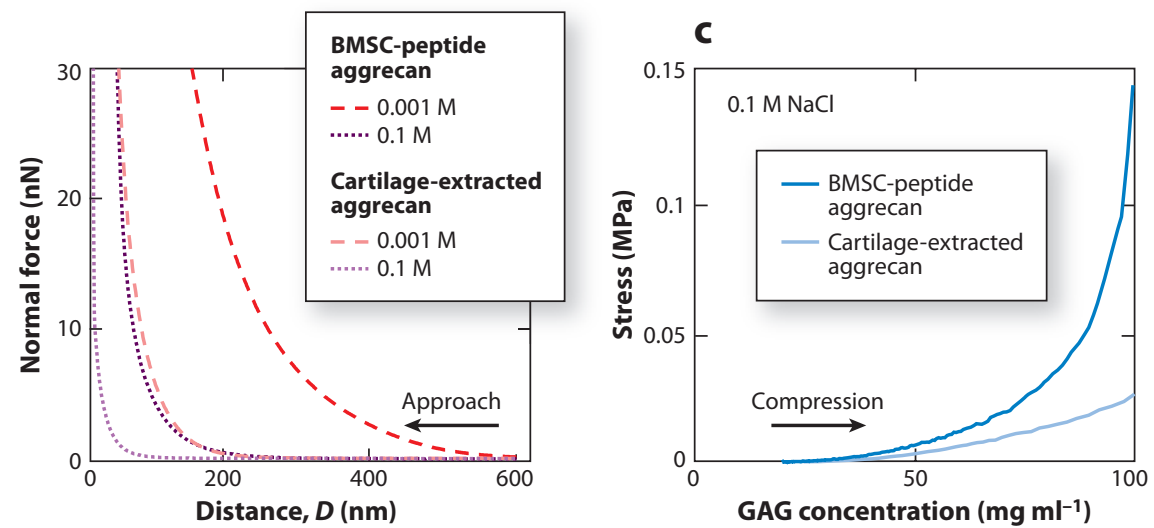

Figure 8

Molecular structure and compressive nanomechanics of tissue-engineered aggrecan. (a) Three-dimensional contact-mode atomic force microscopy (AFM) height image of micropatterned, chemically end-attached aggrecan monolayers in $0.001 \mathrm{M} \mathrm{NaCl}$ aqueous solution at $\sim 100 \mathrm{pN}$ normal load and tapping-mode AFM images of single aggrecan molecules. (Left) Aggrecan synthesized by adult equine bone marrow stromal cells (BMSCs) cultured in self-assembling peptide hydrogel scaffold. (Right) Aggrecan extracted from articular cartilage of adult equine knee joint femoropatellar grooves. (b) (Left) Schematic of compressive nanomechanics experiment on end-grafted aggrecan layer using a neutral hydroxyl-functionalized self-assembling monolayer spherical tip $(R \sim 2.5 \mu \mathrm{m})$. (Right) Corresponding normal force-versus-distance curves in $0.001-1.0 \mathrm{M} \mathrm{NaCl}$ solutions ( $\mathrm{pH} \sim 5.6$ ). (c) Stress-versus-GAG concentration curves converted from the $0.1 \mathrm{M} \mathrm{NaCl}$ data in panel $b$. Adapted with permission from Reference 150.

BMSC aggrecan were more than two times longer than those of adult cartilage aggrecan, which shorten with age $(151,152)$. These distinct structural features may reflect differences in the cell type and cell development; the BMSC aggrecan has a more favorable GAG structure for cartilage mechanical function. Consistent with these structural features, BMSC aggrecan was markedly stiffer in nanomechanical compression than cartilage aggrecan (Figure 8b), even at comparable GAG charge density (Figure 8c). Due to its larger average core protein length and longer GAG chains, the end-grafted BMSC-aggrecan layer also exhibited both greater layer height and larger compressive stiffness (Figure $8 \boldsymbol{b}$ ). Thus, GAG molecular structure and dimensions and GAGGAG electrostatic interactions play an important role in overall aggrecan stiffness (Figure 8c). The more favorable nanostructure and greater stiffness of BMSC aggrecan suggest that this is a promising candidate for cell-based tissue engineering for cartilage repair and regeneration. The combined methodologies utilized here can be applied to optimize the cell source and culture for engineering desired ECM assemblies. 


\subsection{Hyaluronan}

Using SFA, Tadmor et al. (53) assessed the shear forces of free HA in solution and found that the molecules did not adsorb strongly onto mica surfaces and were easily extruded when the separation distance was less than $0.4 \mu \mathrm{m}$. Free HA in synovial fluid is therefore not expected to be a good boundary lubricant, although it may improve hydrodynamic modes of lubrication by contributing to viscosity (53). Results using LFM were consistent with these observations: The presence of free HA in the solution did not reduce the friction coefficient between a spherical probe tip $(R \sim 5 \mu \mathrm{m})$ and a planar substrate functionalized with either hydrophilic (hydroxyl) or hydrophobic (methyl) groups (120).

Tadmor et al. (124) and Benz et al. (125) tested lubrication by HA molecules adsorbed onto mica surfaces in different forms. In the first approach, HA was physically adsorbed onto mica via ion bridging of $\mathrm{Ca}^{2+}$ or by a double layer of octadecyltrimethyl-ammonium bromide and dioctadecyldimethyl-ammonium bromide (124). In the second report, HA was biologically bound to mica by attachment of biotinylated HA to a streptavidin-covered supported lipid bilayer. The latter was proposed as a closer model of articular cartilage (125). In a third report focusing on a lipid bilayer with an inner layer of dipalmitoyl-phosphatidylethanolamine and an outer layer of 95\% dilauroyl-phosphatidylethanolamine and 5\% caproylamine-phosphatidylethanolamine, both linear and cross-linked HA were covalently bound to caproylamine-phosphatidylethanolamine in the presence of a catalyst (Figure 9) (125). The friction coefficients measured for these systems, ranging from 0.15 to $0.4(124,125)$, were much higher than the known friction coefficients of cartilage surfaces (21). Therefore, HA alone is not expected to be responsible for the low friction coefficient of cartilage. However, HA may contribute to cartilage load bearing and wear protection in the form of a surface-grafted network of molecules in the most superficial lamina splendens,

a

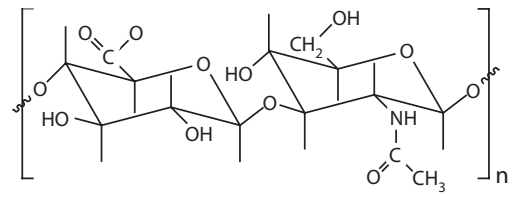

Covalently bonded HA loops

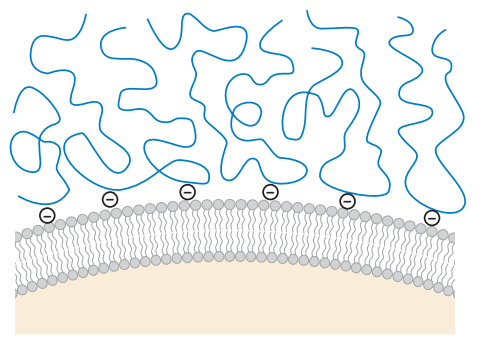

b

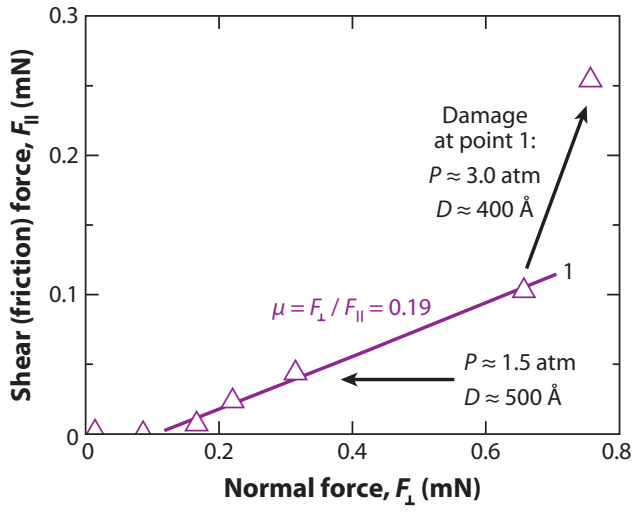

Figure 9

Boundary lubrication by hyaluronan (HA) measured by the surface force apparatus (SFA). (a) Schematic of HA's chemical structure and the proposed mechanism of HA attachment onto a lipid bilayer-coated mica surface: Each HA molecule is expected to covalently attach to positively charged caproylaminephosphatidylethanolamine lipid head groups. (b) Shear (friction) force versus applied normal force between two mica surfaces with covalently attached HA on lipid bilayer coatings (as illustrated in panel $a$ ) in $1 \mathrm{mg}$ $\mathrm{ml}^{-1} \mathrm{HA}$ solution. The friction force increased after damage occurred at point 1 . Adapted with permission from Reference 125 . 
given that cross-linked HA can sustain $\sim 200$ atm pressure before surface damage. HA may be essential for good biolubricity, in conjunction with other biomolecules (125).

\subsection{Lubricin}

The boundary lubrication behavior of lubricin was tested using SFA with adsorbed lubricin layers on negatively charged (mica), hydrophobic (alkanethiol), and positively charged (aminothiol) surfaces (126). On the basis of the chemical components of the two ends of lubricin and the measured normal force profile, the proposed configurations of lubricin molecules on different surfaces are shown in Figure 10a (126). Relatively low friction was observed at forces $>0.4 \mathrm{mN}$ on negatively charged surfaces with free lubricin present in solution. This may be due to hydration layers surrounding the charged polymers, given low brush-brush interpenetration or entanglements upon the formation of a sharp brush-brush interface $(126,153)$. At higher normal force, frictional forces increased when the pressure was above a critical value to induce interpenetration and wear of the brush layers. This lubrication effect was suggested to be related to the negatively charged mucin domain loops and tails because an increase in friction coefficient was observed after digestion (127, $154,155)$ or enzymatic cleavage of the end domains $(127,156)$. This lubrication behavior was absent when lubricin was present in other configurations, e.g., lubricin adsorbed onto hydrophobic or positively charged surfaces (Figure 10b) (126) and enzymatic degraded lubricin (127). Free lubricin in the solution also contributed to the measured lubricity, as suggested by an increase in friction after rinsing (Figure 10b). This observation was consistent with the LFM study (120), in which the presence of lubricin in solution effectively reduced the friction force between two hydrophobic methyl-functionalized surfaces (Figure 10c). The reduction in friction observed with HA and lubricin agreed well with the proposed mechanisms by Klein and coworkers on amphiphilic phospholipids (157) and synthetic, negatively charged polyelectrolytes (153). These mechanisms include the dynamic replacement of water molecules within the hydration sheaths surrounding the charged groups and the resistance to interpenetration for compressed polymer brushes, both of which may occur during cartilage joint lubrication.

\section{MECHANICAL PROPERTIES OF SINGLE MOLECULES}

Several studies have investigated the properties of single ECM macromolecules via SMFS. Haverkamp et al. (114) tested the extensibility of single CS-GAG and HA molecules, together with other connective tissue glycans, in $1 \%$ stock aqueous solutions. They compared the results with the extensible worm-like chain model (Figure 11a) (116). The absence of force-induced conformational transition was observed for both CS-GAG and HA, consistent with their solely equatorial glycosidic bond structure (159). The nanomechanics of the force-induced conformational transitions of pyranose rings may play a role in controlling macroscopic properties of tissues like cartilage. This observation raises the hypothesis that GAG chains may hold ECM collagen fibrils together elastically at strains of up to $10 \%$ (160).

Using SMFS, Harder et al. (115) investigated self-adhesion between a single aggrecan-aggrecan pair in aqueous solution. The observed nonlinear sawtooth pattern, with persistence length $L_{p}=0.31 \pm 0.04 \mathrm{~nm}$, was attributed to the sequential dissociation of individual glycan bonds between the pair of aggrecan molecules (Figure 11b) (115). The presence of $\mathrm{Ca}^{2+}$ provided specific mediation of self-adhesion at the single-molecule level beyond the ion-bridging effect. The mean lifetime $(7.9 \pm 4.9 \mathrm{~s})$ and bond length $(0.31 \pm 0.08 \mathrm{~nm})$ of single-glycan interaction were then estimated by fitting the dissociation rate dependency of self-adhesion to the Bell-Evans model (161). Different from the self-adhesion study on densely packed aggrecan layers that focused on 

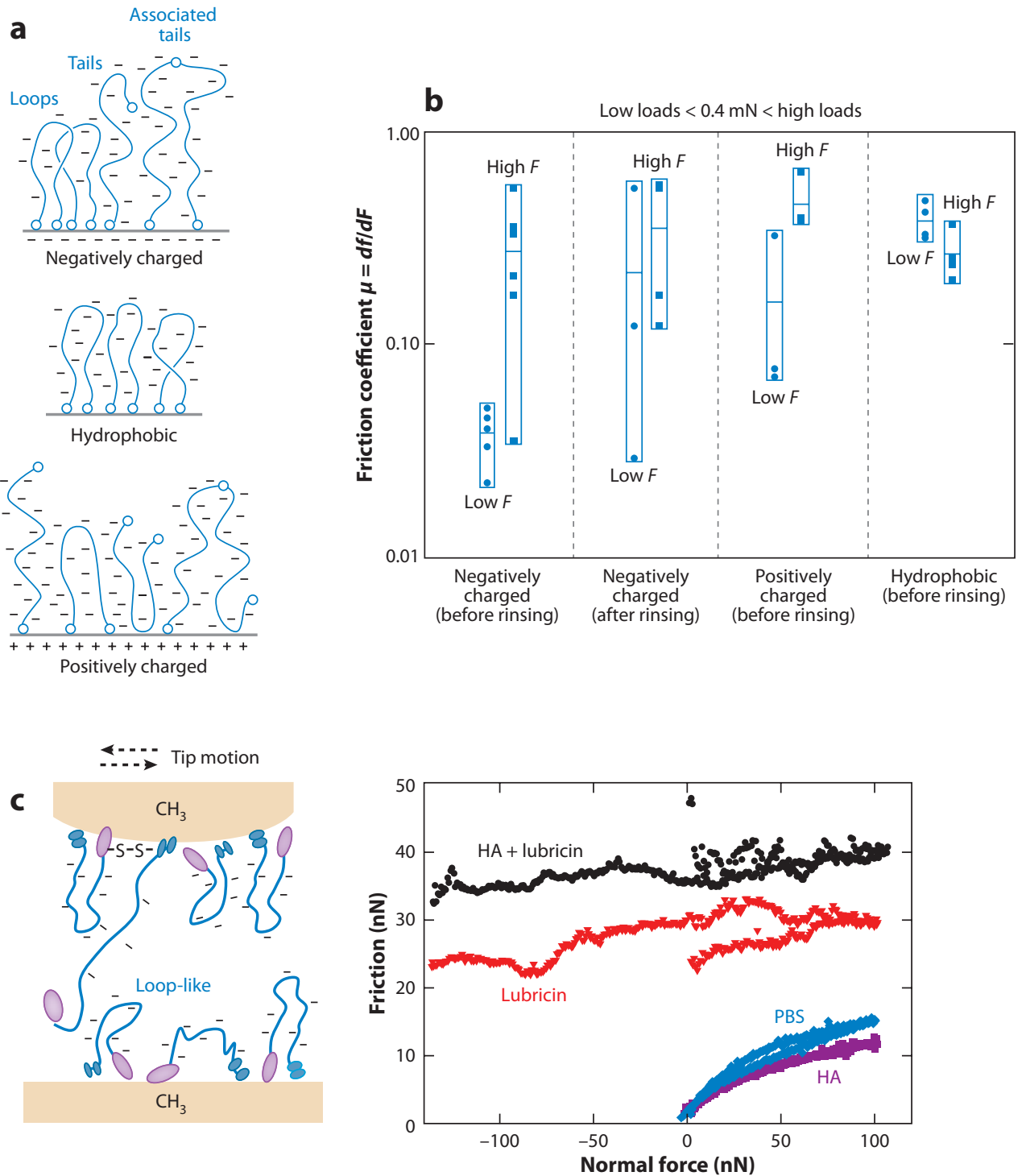

Figure 10

Boundary lubrication by lubricin. (a) Schematic of proposed lubricin physisorbed molecular conformation on mica (negatively charged surface), alkanethiol (hydrophobic surface), and aminothiol (positively charged surface); interlubricin bonding occurs for negatively and positively charged surfaces. (b) Friction coefficients $\mu(=d f / d F$, where $F$ is the applied normal force and $f$ is the friction force) measured for the surfaces shown in panel $a$ in phosphate-buffered saline (PBS) solution of lubricin. Each data point represents an independent experiment. For each group, the horizontal marks indicate the mean values, $\langle\mu\rangle$, and the vertical boxes show the dispersion (scatter) of the experimental data. Panels $a$ and $b$ reproduced with permission from Reference 126. (c) Friction force versus applied normal force between hydroxyl-terminated self-assembled monolayer (OH-SAM) surfaces in PBS, and the addition of $200 \mu \mathrm{g} \mathrm{ml} l^{-1}$ hyaluronan, $3.3 \mathrm{mg} \mathrm{ml}^{-1}$ hyaluronan, and a mixture of lubricin and hyaluronan, as measured via lateral force microscopy using an OH-SAM-functionalized spherical tip $(R \sim 5 \mu \mathrm{m})$. Panel $c$ adapted with permission from Reference 120 . 
a
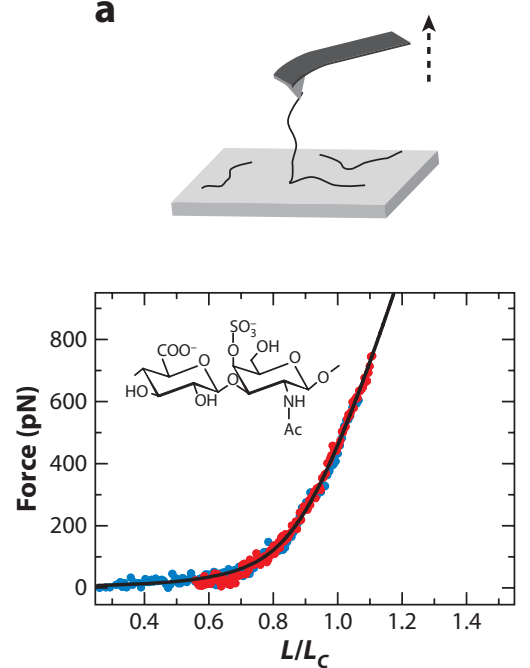

b
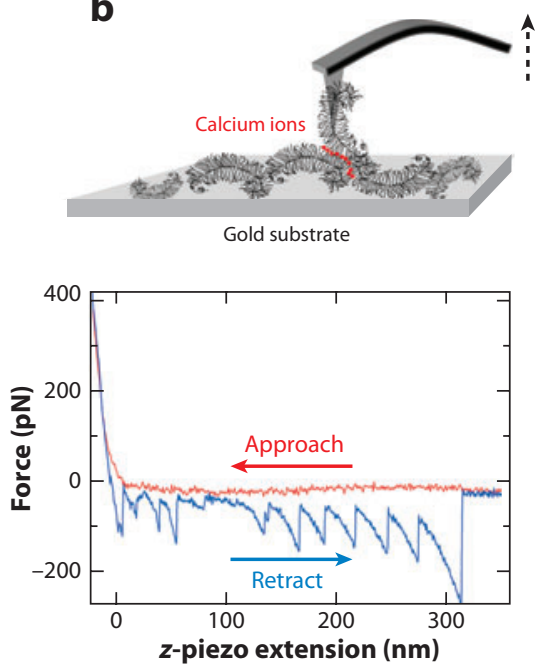

C

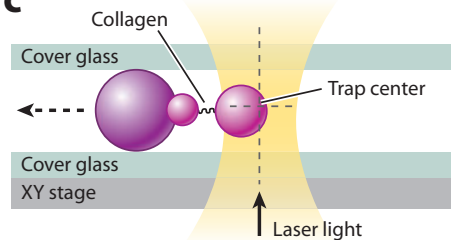

Figure 11

Mechanics of single cartilage extracellular matrix macromolecules. (a) (Top) Schematic of stretching single bovine nasal septal chondroitin sulfate glycosaminoglycan (CS-GAG) in 1\% CS-GAG aqueous solution via atomic force microscopy (AFM)-based single-molecule force spectroscopy (SMFS) (tip radius $R \sim 10 \mathrm{~nm}$ ). (Bottom) Corresponding force-versus-extension curves (displacement normalized to contour length) from the CS-GAG SMFS experiment; the solid line is a fit to the data of the extensible worm-like chain model (116). Data symbols with different colors show multiple repeats of the experiment. (b) (Top) Schematic of calcium-mediated self-adhesion interaction between aggrecan monomers immobilized on a gold-coated tip and gold substrate surface via AFM-based SMFS $(R \sim 25 \mathrm{~nm})$ in $0.1 \mathrm{M} \mathrm{NaCl}+0.01 \mathrm{M} \mathrm{CaCl}_{2}$ aqueous solution (pH 5.6). (Bottom) Corresponding force versus $z$-piezo extension curve from the aggrecan self-adhesion SMFS experiment. (c) (Top) Schematic of stretching a single type II collagen molecule using polystyrene beads $(R \sim 2.05 \mu \mathrm{m})$ via optical tweezers. The collagen is stretched as the large bead is moved away from the trapping center of the XY stage in the aqueous solution of $25 \mathrm{mM} \mathrm{KCl}, 1 \mathrm{mM} \mathrm{EGTA}, 0.2 \%$ Tween $20,0.1 \%$ casein, and $25 \mathrm{mM}$ HEPES at $\mathrm{pH}$ 7.4. (Bottom) Corresponding force-versus-extension curve from the type II collagen extension optical tweezers experiment; the solid line is a fit to the data of the extensible worm-like chain model (116). The contour length $L_{c}$ and persistence length $L_{p}$ are $300 \mathrm{~nm}$ and $7.6 \mathrm{~nm}$, respectively. Panels $a, b$, and $c$ adapted with permission from References 114, 115, and 130, respectively.

physiologically relevant interactions (145), this study probed single-molecule interactions. The estimated parameters were thought to provide a linkage between the single-molecular to macroscopic tissue properties, such as the intrinsic viscoelasticity.

Optical tweezers have been applied to directly measure the extensibility of single HA (129) and type II collagen (130) (Figure 11c) molecules at higher force resolution. For single HA molecules, the force-displacement relationship was similar to that observed via SMFS, and an average persistence length of $L_{p} \sim 4.4-4.5 \mathrm{~nm}$ over contour length $L_{c} \sim 2.6 \mu \mathrm{m}$ was calculated on the basis of extensible worm-like chain models. This high flexibility of HA was consistent with its cartilage tissue function as the backbone of aggrecan aggregates to absorb compressive loads, whereas tensile loads are carried mainly by the collagen fibrils. Interestingly, single procollagen II (a precursor to the type II collagen molecule within fibrils; Figure 11c) was measured to be very flexible as well, with $L_{p} \sim 7.6 \mathrm{~nm}$ over $L_{c} \sim 300 \mathrm{~nm}$. Such flexibility may be important for the secretion of procollagen molecules into the ECM for collagen fibril self-assembly and interaction with other molecules, as well as for cell attachment (130). In comparison, aggrecan monomers $\left(L_{p} \sim 80-110 \mathrm{~nm}\right)$ and their CS-GAG branches $\left(L_{p} \sim 14-21 \mathrm{~nm}\right)$ showed much higher stiffness, as assessed by their conformation using tapping-mode AFM (40), consistent with their tissue function as the major determinants of cartilage swelling pressure and compressive stiffness. 


\section{CONCLUSIONS AND FUTURE OUTLOOK}

Historically, cartilage research has involved macroscopic mechanical testing and biochemical assays that provide homogenized and averaged information from large populations of molecules and cells. However, the detailed and spatially heterogeneous hierarchical structure, composition, and properties of cartilage are critical to its biomechanical and biophysical function and dysfunction. The field of nanomechanics has opened the door to a new understanding of the underlying mechanistic origins of tissue-level behavior and will be important to the development and improvement of molecular-based therapeutics for joint disease and tissue engineering for cartilage defect repair. This review summarizes significant progress in the following areas:

- Spatial heterogeneity. Micrometer- and nanometer-scale spatial heterogeneity (e.g. PCM, territorial, interterritorial matrices, depth-dependent zones) of biomechanical and biophysical properties has been detected and quantified. Further investigations are needed to understand the role of spatial heterogeneity in the nanoscale function and dysfunction of cartilage. Such high-resolution localized experiments may have potential for early diagnostics.

- Small tissue volumes. The biomechanical and biophysical effects of aging, disease, and gene mutations/deletions can now be quantified in small and irregular volumes of tissue, thereby enabling the direct quantification of biomechanical properties on important mouse models subjected to gene deletion of specific cartilage matrix molecules, such as fibromodulin and chondroadherin.

- Molecular origins of macroscopic mechanical bebavior. The nonlinear repulsive electrostatic and nonelectrostatic interactions between GAGs and aggrecan are critical factors in determining cartilage tissue-level nonlinear strain-hardening (self-stiffening) compressive and shear behavior. Aggrecan self-adhesion has been detected and quantified and likely contributes to the structural integrity of the tissue.

- Quality of tissue-engineered cartilage. Ultrastructure and nanomechanical properties of tissueengineered cartilage associated with individual cells can be quantified at the nanoscale and provide an invaluable high-resolution assessment for optimizing design principles for cartilage tissue engineering and repair.

There are a number of future opportunities for the application of nanomechanics to cartilage and other biological tissues. (a) New advances in multiscale modeling connecting atomistics to the molecular level to the mesoscale to the microscale to the macroscale will eventually close the knowledge gap on how each structural level contributes to the homogenized macroscopic response and what role they play independently in the function and degradation of the tissue via a variety of coarse-graining methods, such as single-bead models, elastic-like models, and the GO model (171). (b) A combination of HRFS, fluorescence microscopy, and biochemical readouts may enable a new field of single-cell mechanobiology. With the recent advances in detecting gene expression of single cells using subnanoliter wells (172) and discovering monoclonal biomolecular antibody using microengraving (173), these methods can be utilized to quantify the biochemical response of single cells as they are subjected to different loading and strain conditions. (c) A third emerging area involves probing nanomechanical and physical processes in situ using transmission and scanning electron microscopy, wherein both material properties and specimen structure can be monitored simultaneously during loading $(174,175)$. Unfortunately, this technique is not applicable to cartilage yet because cartilage mechanical testing needs to be conducted in aqueous solutions for physiological relevance, whereas electron microscopy requires vacuum conditions. However, recent developments in cryo-stage focused ion beam techniques (176) may be suitable candidates for the in situ nanoscale/microscale mechanical measurements of biological systems such as cartilage. 


\section{DISCLOSURE STATEMENT}

The authors are not aware of any affiliations, memberships, funding, or financial holdings that might be perceived as affecting the objectivity of this review.

\section{ACKNOWLEDGMENTS}

The authors thank the Institute for Soldier Nanotechnologies at MIT, funded through the U.S. Army Research Office, for use of instruments. This work was supported by the National Science Foundation (grant CMMI-0758651), the National Institute of Health (Grants AR033236 and EB003805), and the National Security Science and Engineering Faculty Fellowship (grant N00244-09-1-0064).

\section{LITERATURE CITED}

1. Cleland AN. 2002. Foundations of Nanomechanics: From Solid-State Theory to Device Applications. New York: Springer

2. Lin DC, Horkay F. 2008. Nanomechanics of polymer gels and biological tissues: a critical review of analytical approaches in the Hertzian regime and beyond. Soft Matter 4:669-82

3. Leckband D, Israelachvili J. 2001. Intermolecular forces in biology. Q. Rev. Biophys. 34:105-267

4. Israelachvili JN. 1992. Intermolecular and Surface Forces. London: Academic

5. Bao G, Suresh S. 2003. Cell and molecular mechanics of biological materials. Nat. Mater. 2:715-25

6. Neuman KC, Nagy A. 2008. Single-molecule force spectroscopy: optical tweezers, magnetic tweezers and atomic force microscopy. Nat. Methods 5:491-505

7. Sakamoto J, Decuzzi P, Gentile F, Rokhlin SI, Wang L, et al. 2006. Nanomechanics and tissue pathology. In BioMEMS and Biomedical Nanotechnology. Vol. 1. Biological and Biomedical Nanotechnology, ed. AP Lee, LJ Lee, M Ferrari, pp. 461-503. New York: Springer

8. Aigner T, Schmitz N, Haag J. 2009. Nanomedicine: AFM tackles osteoarthritis. Nat. Nanotechnol. 4:14445

9. Hunziker PR, Stolz M, Aebi U. 2002. Nanotechnology in medicine: moving from the bench to the bedside. Chimia 56:520-26

10. Stolz M, Aebi U, Stoffler D. 2007. Developing scanning probe-based nanodevices: stepping out of the laboratory into the clinic. Nanomed. Nanotechnol. Biol. Med. 3:53-62

11. Ng L, Hung H-H, Sprunt A, Chubinskaya S, Ortiz C, Grodzinsky AJ. 2007. Nanomechanical properties of individual chondrocytes and their developing growth factor-stimulated pericellular matrix. F. Biomech. 40:1011-23

12. Lee B, Han L, Frank EH, Chubinskaya S, Ortiz C, Grodzinsky AJ. 2010. Dynamic mechanical properties of the tissue-engineered matrix associated with individual chondrocytes. 7. Biomech. 43:469-76

13. Tai K, Pelled G, Sheyn D, Bershteyn A, Han L, et al. 2008. Nanobiomechanics of repair bone regenerated by genetically modified mesenchymal stem cells. Tissue Eng. A 14:1709-20

14. Fortina P, Kricka LJ. 2010. Nanotechnology: improving clinical testing? Clin. Chem. 56:1384-89

15. Naujoks C, Meyer U, Wiesmann H-P, Jasche-Meyer J, Hohoff A, et al. 2008. Principles of cartilage tissue engineering in TMJ reconstruction. Head Face Med. 4:3

16. Kuo CK, Li WJ, Mauck RL, Tuan RS. 2006. Cartilage tissue engineering: its potential and uses. Curr. Opin. Rheumatol. 18:64-73

17. Freeman MAR, ed. 1979. Adult Articular Cartilage. Kent: Pitman Med.

18. Maroudas A. 1979. Physicochemical properties of articular cartilage. See Ref. 17, pp. 215-90

19. Morrell KC, Hodge WA, Krebs DE, Mann RW. 2005. Corroboration of in vivo cartilage pressures with implications for synovial joint tribology and osteoarthritis causation. Proc. Natl. Acad. Sci. USA 102:14819-24

20. Van de Velde SK, Bingham JT, Hosseini A, Kozanek M, DeFrate LE, et al. 2009. Increased tibiofemoral cartilage contact deformation in patients with anterior cruciate ligament deficiency. Arthritis Rheum. 60:3693-702 
21. Forster H, Fisher J. 1996. The influence of loading time and lubricant on the friction of articular cartilage. Proc. Inst. Mech. Eng. H 210:109-19

22. Bonassar LJ, Jeffries KA, Paguio CG, Grodzinsky AJ. 1995. Cartilage degradation and associated changes in biomechanical and electromechanical properties. Acta Orthop. Scand. Suppl. 66:38-44

23. Lee RC, Frank EH, Grodzinsky AJ, Roylance DK. 1981. Oscillatory compressional behavior of articular cartilage and its associated electromechanical properties. F. Biomech. Eng. 103:280-92

24. Kim Y-J, Bonassar LJ, Grodzinsky AJ. 1995. The role of cartilage streaming potential, fluid flow and pressure in the stimulation of chondrocyte biosynthesis during dynamic compression. F. Biomech. 28:1055-66

25. Hayes WC, Bodine AJ. 1978. Flow-independent viscoelastic properties of articular cartilage matrix. 7. Biomech. 11:407-19

26. Jin M, Grodzinsky AJ. 2001. Effect of electrostatic interactions between glycosaminoglycans on the shear stiffness of cartilage: a molecular model and experiments. Macromolecules 34:8330-39

27. Grodzinsky AJ, Roth V, Myers E, Grossman WD, Mow VC. 1981. The significance of electromechanical and osmotic forces in the nonequilibrium swelling behavior of articular cartilage in tension. 7. Biomech. Eng. 103:221-31

28. Kempson GE, Freeman MAR, Swanson SAV. 1971. The determination of a creep modulus for articular cartilage from indentation tests on the human femoral head. F. Biomech. 4:239-50

29. Mow VC, Gibbs MC, Lai WM, Zhu WB, Athanasiou KA. 1989. Biphasic indentation of articular cartilage. II. A numerical algorithm and an experimental study. 7. Biomech. 22:853-61

30. Mak AF. 1986. The apparent viscoelastic behavior of articular cartilage: the contributions from the intrinsic matrix viscoelasticity and interstitial fluid flows. F. Biomech. Eng. 108:123-30

31. Mow VC, Kuei SC, Lai WM, Armstrong CG. 1980. Biphasic creep and stress relaxation of articular cartilage in compression: theory and experiments. F. Biomech. Eng. 102:73-84

32. Muir IHM. 1979. Biochemistry. See Ref. 17, pp. 145-214

33. Stockwell RA, Meachim G. 1979. The chondrocytes. See Ref. 17, pp. 69-144

34. Hunziker EB, Kapfinger E, Geiss J. 2007. The structural architecture of adult mammalian articular cartilage evolves by a synchronized process of tissue resorption and neoformation during postnatal development. Osteoarthr. Cartil. 15:403-13

35. Heinegard D. 2009. Proteoglycans and more: from molecules to biology. Int. 7. Exp. Pathol. 90:575-86

36. Kopesky PW, Lee H-Y, Vanderploeg EJ, Kisiday JD, Frisbie DD, et al. 2010. Adult equine bone marrow stromal cells produce a cartilage-like ECM mechanically superior to animal-matched adult chondrocytes. Matrix Biol. 29:427-38

37. Eyre D. 2002. Collagen of articular cartilage. Arthritis Res. 4:30-35

38. Meachim G, Stockwell RA. 1979. The matrix. See Ref. 17, pp. 1-68

39. Rosenberg L, Hellmann W, Kleinschmidt AK. 1970. Macromolecular models of proteinpolysaccharides from bovine nasal cartilage based on electron microscopic studies. 7. Biol. Chem. 245:4123-30

40. Ng L, Grodzinsky AJ, Patwari P, Sandy J, Plaas A, Ortiz C. 2003. Individual cartilage aggrecan macromolecules and their constituent glycosaminoglycans visualized via atomic force microscopy. F. Struct. Biol. 143:242-57

41. Hardingham TE, Muir H. 1972. The specific interaction of hyaluronic acid with cartilage proteoglycans. Biochim. Biophys. Acta 279:401-5

42. Maroudas A, Mizrahi J, Ben Haim E, Ziv I. 1987. Swelling pressure in cartilage. Adv. Microcirc. 13:203-12

43. Sokoloff L, ed. 1978-1980. The foints and Synovial Fluid. London: Academic

44. Maroudas A. 1980. Physical chemistry of articular cartilage and the intervertebral disk in joints and synovial fluid. See Ref. 43, Vol. 2, pp. 240-93

45. Williamson AK, Chen AC, Sah RL. 2001. Compressive properties and function-composition relationships of developing bovine articular cartilage. F. Orthop. Res. 19:1113-21

46. Zhu W, Mow VC, Koob TJ, Eyre DR. 1993. Viscoelastic shear properties of articular cartilage and the effects of glycosidase treatments. F. Orthop. Res. 11:771-81

47. Sandy JD, Neame PJ, Boynton RE, Flannery CR. 1991. Catabolism of aggrecan in cartilage explants. Identification of a major cleavage site within the interglobular domain. F. Biol. Chem. 266:8683-85

48. Lark MW, Bayne EK, Lohmander LS. 1995. Aggrecan degradation in osteoarthritis and rheumatoid arthritis. Acta Orthop. Scand. Suppl. 266:92-97 
49. Heinegard D, Saxne T. 2011. The role of the cartilage matrix in osteoarthritis. Nat. Rev. Rheumatol. 7:50-56

50. Cowman MK, Li M, Balazs EA. 1998. Tapping mode atomic force microscopy of hyaluronan: extended and intramolecularly interacting chains. Biophys. 7. 75:2030-37

51. Mikecz K, Brennan FR, Kim JH, Glant TT. 1995. Anti-CD44 treatment abrogates tissue oedema and leukocyte infiltration in murine arthritis. Nat. Med. 1:558-63

52. McCutchen CW. 1978. Lubrication of joints. See Reference 43, Vol. 1, pp. 437-83

53. Tadmor R, Chen N, Israelachvili JN. 2002. Thin film rheology and lubricity of hyaluronic acid solutions at a normal physiological concentration. 7. Biomed. Mater. Res. 61:514-23

54. Howard RD, McIlwraith CW. 1993. Sodium hyaluronate in the treatment of equine joint disease. Compend. Contin. Ed. Pract. Vet. 15:473-79

55. Hills BA. 2000. Boundary lubrication in vivo. Proc. Inst. Mech. Eng. H 214:83-94

56. Mori S, Naito M, Moriyama S. 2002. Highly viscous sodium hyaluronate and joint lubrication. Int. Orthop. 26:116-21

57. Jay GD. 2004. Lubricin and surfacing of articular joints. Curr. Opin. Orthop. 15:355-59

58. Radin EL, Swann DA, Weisser PA. 1970. Separation of a hyaluronate-free lubricating fraction from synovial fluid. Nature 228:377-78

59. Swann DA, Slayter HS, Silver FH. 1981. The molecular structure of lubricating glycoprotein-I, the boundary lubricant for articular cartilage. F. Biol. Chem. 256:5921-25

60. Jay GD, Britt DE, Cha CJ. 2000. Lubricin is a product of megakaryocyte stimulating factor gene expression by human synovial fibroblasts. F. Rheumatol. 27:594-600

61. Rhee DK, Marcelino J, Baker MA, Gong Y, Smits P, et al. 2005. The secreted glycoprotein lubricin protects cartilage surfaces and inhibits synovial cell overgrowth. F. Clin. Investig. 115:622-31

62. Flannery CR, Zollner R, Corcoran C, Jones AR, Root A, et al. 2009. Prevention of cartilage degeneration in a rat model of osteoarthritis by intraarticular treatment with recombinant lubricin. Artbritis Rheum. 60:840-47

63. Wiberg C, Hedbom E, Khairullina A, Lamande SR, Oldberg A, et al. 2001. Biglycan and decorin bind close to the N-terminal region of the collagen VI triple helix. F. Biol. Chem. 276:18947-52

64. Wiberg C, Klatt AR, Wagener R, Paulsson M, Bateman JF, et al. 2003. Complexes of matrilin-1 and biglycan or decorin connect collagen VI microfibrils to both collagen II and aggrecan. 7. Biol. Chem. 278:37698-704

65. Hedbom E, Heinegard D. 1993. Binding of fibromodulin and decorin to separate sites on fibrillar collagens. 7. Biol. Chem. 268:27307-12

66. Kalamajski S, Oldberg A. 2009. Homologous sequence in lumican and fibromodulin leucine-rich repeat 5-7 competes for collagen binding. 7. Biol. Chem. 284:534-39

67. Kalamajski S, Aspberg A, Lindblom K, Heinegard D, Oldberg A. 2009. Asporin competes with decorin for collagen binding, binds calcium and promotes osteoblast collagen mineralization. Biochem. 7 . 423:5359

68. Halasz K, Kassner A, Morgelin M, Heinegard D. 2007. COMP acts as a catalyst in collagen fibrillogenesis. 7. Biol. Chem. 282:31166-73

69. Camper L, Heinegard D, Lundgren-Akerlund E. 1997. Integrin $\alpha 2 \beta 1$ is a receptor for the cartilage matrix protein chondroadherin. F. Cell Biol. 138:1159-67

70. Hardingham TE, Fosang AJ. 1992. Proteoglycans: many forms and many functions. FASEB 7. 6:861-70

71. Buschmann MD, Grodzinsky AJ. 1995. A molecular model of proteoglycan-associated electrostatic forces in cartilage mechanics. 7. Biomech. Eng. 117:179-92

72. Bathe M, Rutledge GC, Grodzinsky AJ, Tidor B. 2005. A coarse-grained molecular model for glycosaminoglycans: application to chondroitin, chondroitin sulfate, and hyaluronic acid. Biophys. 7 . 88:3870-87

73. Bathe M, Rutledge GC, Grodzinsky A, Tidor B. 2005. Osmotic pressure of aqueous chondroitin sulfate solution: a molecular modeling investigation. Biophys. F. 89:2357-71

74. Nap RJ, Szleifer I. 2008. Structure and interactions of aggrecans: statistical thermodynamic approach. Biophys. 7. 95:4570-83 
75. Reynaud B, Quinn TM. 2006. Anisotropic hydraulic permeability in compressed articular cartilage. 7. Biomech. 39:131-37

76. Eisenberg SR, Grodzinsky AJ. 1988. Electrokinetic micromodel of extracellular matrix and other polyelectrolyte networks. PhysicoChem. Hydrodyn. 10:517-39

77. Guilak F, Ratcliffe A, Mow VC. 1995. Chondrocyte deformation and local tissue strain in articular cartilage: a confocal microscopy study. F. Orthop. Res. 13:410-21

78. Radmacher M. 1997. Measuring the elastic properties of biological samples with the AFM. IEEE Eng. Med. Biol. Mag. 16:47-57

79. Seog J, Dean D, Plaas AHK, Wong-Palms S, Grodzinsky AJ, Ortiz C. 2002. Direct measurement of glycosaminoglycan intermolecular interactions via high-resolution force spectroscopy. Macromolecules 35:5601-15

80. Takano H, Kenseth JR, Wong S-S, O’Brien JC, Porter MD. 1999. Chemical and biochemical analysis using scanning force microscopy. Chem. Rev. 99:2845-90

81. VanLandingham MR. 2003. Review of instrumented indentation. 7. Res. Natl. Inst. Stand. Technol. 108:249-65

82. Israelachvili JN, Adams GE. 1976. Direct measurement of long range forces between two mica surfaces in aqueous $\mathrm{KNO}_{3}$ solutions. Nature 262:774-76

83. Klein J. 1980. Forces between mica surfaces bearing layers of adsorbed polystyrene in cyclohexane. Nature 288:248-50

84. Oliver WC, Pharr GM. 1992. An improved technique for determining hardness and elastic modulus using load and displacement sensing indentation experiments. F. Mater. Res. 7:1564-83

85. Loening AM, James IE, Levenston ME, Badger AM, Frank EH, et al. 2000. Injurious mechanical compression of bovine articular cartilage induces chondrocyte apoptosis. Arch. Biochem. Biophys. 381:20512

86. Miller GJ, Morgan EF. 2010. Use of microindentation to characterize the mechanical properties of articular cartilage: comparison of biphasic material properties across length scales. Osteoarthr. Cartil. 18:1051-57

87. Lewis JL, Johnson SL. 2001. Collagen architecture and failure processes in bovine patellar cartilage. 7. Anat. $199: 483-92$

88. Hertz H. 1881. Über die Berührung fester elastischer Körpen (On the contact of rigid elastic solids). 7 . R. Angew. Math. 92:156-71

89. Johnson KL, Kendall K, Roberts AD. 1971. Surface energy and the contact of elastic solids. Proc. R. Soc. Lond. Ser. A 324:301-13

90. Derjaguin BV, Muller VM, Toporov YP. 1975. Effect of contact deformations on the adhesion of particles. 7. Colloid Interface Sci. 53:314-26

91. Stolz M, Raiteri R, Daniels AU, VanLandingham MR, Baschong W, Aebi U. 2004. Dynamic elastic modulus of porcine articular cartilage determined at two different levels of tissue organization by indentation-type atomic force microscopy. Biophys. 7. 86:3269-83

92. Buschmann MD, Kim Y-J, Wong M, Frank EH, Hunziker EB, Grodzinsky AJ. 1999. Stimulation of aggrecan synthesis in cartilage explants by cyclic loading is localized to regions of high interstitial fluid flow. Arch. Biochem. Biophys. 366:1-7

93. Bilodeau GG. 1992. Regular pyramid punch problem. F. Appl. Mech. 59:519-23

94. Gupta S, Lin J, Ashby P, Pruitt L. 2009. A fiber reinforced poroelastic model of nanoindentation of porcine costal cartilage: a combined experimental and finite element approach. F. Mech. Behav. Biomed. Mater. 2:326-38

95. Lin DC, Dimitriadis EK, Horkay F. 2007. Robust strategies for automated AFM force curve analysis. I. Non-adhesive indentation of soft, inhomogeneous materials. F. Biomech. Eng. 129:430-40

96. Stolz M, Gottardi R, Raiteri R, Miot S, Martin I, et al. 2009. Early detection of aging cartilage and osteoarthritis in mice and patient samples using atomic force microscopy. Nat. Nanotechnol. 4:186-92

97. Loparic M, Wirz D, Daniels AU, Raiteri R, VanLandingham MR, et al. 2010. Micro- and nanomechanical analysis of articular cartilage by indentation-type atomic force microscopy: validation with a gel-microfiber composite. Biophys. 7. 98:2731-40 
98. Jurvelin JS, Muller DJ, Wong M, Studer D, Engel A, Hunziker EB. 1996. Surface and subsurface morphology of bovine humeral articular cartilage as assessed by atomic force and transmission electron microscopy. 7. Struct. Biol. 117:45-54

99. Hu K, Radhakrishnan P, Patel RV, Mao JJ. 2001. Regional structural and viscoelastic properties of fibrocartilage upon dynamic nanoindentation of the articular condyle. F. Struct. Biol. 136:46-52

100. Radhakrishnan P, Lewis NT, MaoJJ. 2004. Zone-specific micromechanical properties of the extracellular matrices of growth plate cartilage. Ann. Biomed. Eng. 32:284-91

101. Patel RV, Mao JJ. 2003. Microstructural and elastic properties of the extracellular matrices of the superficial zone of neonatal articular cartilage by atomic force microscopy. Front. Biosci. 8:A18-25

102. Tomkoria S, Patel RV, Mao JJ. 2004. Heterogeneous nanomechanical properties of superficial and zonal regions of articular cartilage of the rabbit proximal radius condyle by atomic force microscopy. Med. Eng. Pbys. 26:815-22

103. Allen DM, Mao JJ. 2004. Heterogeneous nanostructural and nanoelastic properties of pericellular and interterritorial matrices of chondrocytes by atomic force microscopy. F. Struct. Biol. 145:196-204

104. Park S, Costa KD, Ateshian GA, Hong K-S. 2009. Mechanical properties of bovine articular cartilage under microscale indentation loading from atomic force microscopy. Proc. Inst. Mech. Eng. H 223:339-47

105. Darling EM, Wilusz RE, Bolognesi MP, Zauscher S, Guilak F. 2010. Spatial mapping of the biomechanical properties of the pericellular matrix of articular cartilage measured in situ via atomic force microscopy. Biophys. 7. 98:2848-56

106. Ebenstein DM, Kuo A, Rodrigo JJ, Reddi AH, Ries M, Pruitt L. 2004. A nanoindentation technique for functional evaluation of cartilage repair tissue. 7. Mater. Res. 19:273-81

107. Li C, Pruitt LA, King KB. 2006. Nanoindentation differentiates tissue-scale functional properties of native articular cartilage. 7. Biomed. Mater. Res. A 78:729-38

108. Gupta HS, Schratter S, Tesch W, Roschger P, Berzlanovich A, et al. 2005. Two different correlations between nanoindentation modulus and mineral content in the bone-cartilage interface. 7 . Struct. Biol. 149:138-48

109. Franke O, Durst K, Maier V, Goken M, Birkholz T, et al. 2007. Mechanical properties of hyaline and repair cartilage studied by nanoindentation. Acta Biomater. 3:873-81

110. Dean D, Han L, Ortiz C, Grodzinsky AJ. 2005. Nanoscale conformation and compressibility of cartilage aggrecan using microcontact printing and atomic force microscopy. Macromolecules 38:4047-49

111. Seog J, Dean D, Rolauffs B, Wu T, Genzer J, et al. 2005. Nanomechanics of opposing glycosaminoglycan macromolecules. F. Biomech. 38:1789-97

112. Dean D, Han L, Grodzinsky AJ, Ortiz C. 2006. Compressive nanomechanics of opposing aggrecan macromolecules. F. Biomech. 39:2555-65

113. Dean D, Seog J, Ortiz C, Grodzinsky AJ. 2003. Molecular-level theoretical model for electrostatic interactions within polyelectrolyte brushes: applications to charged glycosaminoglycans. Langmuir 19:552639

114. Haverkamp RG, Williams MA, Scott JE. 2005. Stretching single molecules of connective tissue glycans to characterize their shape-maintaining elasticity. Biomacromolecules 6:1816-18

115. Harder A, Walhorn V, Dierks T, Xavier F-B, Anselmetti D. 2010. Single-molecule force spectroscopy of cartilage aggrecan self-adhesion. Biophys. F. 99:3498-504

116. Wang MD, Yin H, Landick R, Gelles J, Block SM. 1997. Stretching DNA with optical tweezers. Biophys. 7. $72: 1335-46$

117. Ogletree DF, Carpick RW, Salmeron M. 1996. Calibration of frictional forces in atomic force microscopy. Rev. Sci. Instrum. 67:3298-306

118. Park S, Costa KD, Ateshian GA. 2004. Microscale frictional response of bovine articular cartilage from atomic force microscopy. F. Biomech. 37:1679-87

119. Chan SMT, Neu CP, DuRaine G, Komvopoulos K, Reddi AH. 2010. Atomic force microscope investigation of the boundary-lubricant layer in articular cartilage. Osteoarthr. Cartil. 18:956-63

120. Chang DP, Abu-Lail NI, Coles JM, Guilak F, Jay GD, Zauscher S. 2009. Friction force microscopy of lubricin and hyaluronic acid between hydrophobic and hydrophilic surfaces. Soft Matter 5:3438-45

121. Wilbur JL, Kumar A, Kim E, Whitesides GM. 1994. Microfabrication by microcontact printing of self-assembled monolayers. Adv. Mater. 6:600-4 
122. Han L, Dean D, Ortiz C, Grodzinsky AJ. 2007. Lateral nanomechanics of cartilage aggrecan macromolecules. Biophys. 7. 92:1384-98

123. Han L, Dean D, Mao P, Ortiz C, Grodzinsky AJ. 2007. Nanoscale shear deformation mechanisms of opposing cartilage aggrecan macromolecules. Biophys. 7. 93:L23-25

124. Tadmor R, Chen N, Israelachvili J. 2003. Normal and shear forces between mica and model membrane surfaces with adsorbed hyaluronan. Macromolecules 36:9519-26

125. Benz M, Chen N, Israelachvili J. 2004. Lubrication and wear properties of grafted polyelectrolytes, hyaluronan and hylan, measured in the surface forces apparatus. F. Biomed. Mater. Res. A 71:6-15

126. Zappone B, Ruths M, Greene GW, Jay GD, Israelachvili JN. 2007. Adsorption, lubrication, and wear of lubricin on model surfaces: polymer brush-like behavior of a glycoprotein. Biophys. 7. 92:1693-708

127. Zappone B, Greene GW, Oroudjev E, Jay GD, Israelachvili JN. 2008. Molecular aspects of boundary lubrication by human lubricin: effect of disulfide bonds and enzymatic digestion. Langmuir 24:1495-508

128. Ashkin A. 1970. Acceleration and trapping of particles by radiation pressure. Phys. Rev. Lett. 24:156-59

129. Fujii T, Sun Y-L, An K-N, Luo Z-P. 2002. Mechanical properties of single hyaluronan molecules. 7. Biomech. 35:527-31

130. Sun Y-L, Luo Z-P, Fertala A, An K-N. 2004. Stretching type II collagen with optical tweezers. F. Biomech. 37:1665-69

131. Liu X, Noble PC, Luo Z-P. 2003. A method for testing compressive properties of single proteoglycan aggregates. Biochem. Biophys. Res. Commun. 307:338-41

132. O'Connor KM. 1997. Unweighting accelerates tidemark advancement in articular cartilage at the knee joint of rats. 7. Bone Miner. Res. 12:580-89

133. Poole CA. 1997. Articular cartilage chondrons: form, function and failure. F. Anat. 191:1-13

134. Alexopoulos LG, Setton LA, Guilak F. 2005. The biomechanical role of the chondrocyte pericellular matrix in articular cartilage. Acta Biomater. 1:317-25

135. Helminen HJ, Saamanen A-M, Salminen H, Hyttinen MM. 2002. Transgenic mouse models for studying the role of cartilage macromolecules in osteoarthritis. Rheumatology 41:848-56

136. Han L, Plaas AHK, Sandy JD, Frank EH, Hung H-HK, et al. 2009. Nanomechanics of murine knee joints reveals effects of maturation and inflammation. Trans. Orthop. Res. Soc. 55:324

137. Chang J, Poole CA. 1997. Confocal analysis of the molecular heterogeneity in the pericellular microenvironment produced by adult canine chondrocytes cultured in agarose gel. Histochem. F. 29:515-28

138. Eyre DR, Apon S, Wu J-J, Ericsson LH, Walsh KA. 1987. Collagen type IX: evidence for covalent linkages to type II collagen in cartilage. FEBS Lett. 220:337-41

139. Poole CA, Flint MH, Beaumont BW. 1988. Chondrons extracted from canine tibial cartilage: preliminary report on their isolation and structure. F. Orthop. Res. 6:408-19

140. Buschmann MD, Gluzband YA, Grodzinsky AJ, Kimura JH, Hunziker EB. 1992. Chondrocytes in agarose culture synthesize a mechanically functional extracellular matrix. F. Orthop. Res. 10:745-58

141. Mauck RL, Soltz MA, Wang CCB, Wong DD, Chao P-HG, et al. 2000. Functional tissue engineering of articular cartilage through dynamic loading of chondrocyte-seeded agarose gels. F. Biomech. Eng. $122: 252-60$

142. DiMicco MA, Kisiday JD, Gong H, Grodzinsky AJ. 2007. Structure of pericellular matrix around agarose-embedded chondrocytes. Osteoarthr. Cartil. 15:1207-16

143. Coles JM, Zhang L, Blum JJ, Warman ML, Jay GD, et al. 2010. Loss of cartilage structure, stiffness, and frictional properties in mice lacking PRG4. Arthritis Rheum. 62:1666-74

144. Seog J, Dean D, Frank EH, Ortiz C, Grodzinsky AJ. 2004. Preparation of end-grafted polyelectrolytes on nanoscale probe tips using an electric field. Macromolecules 37:1156-58

145. Han L, Dean D, Daher LA, Grodzinsky AJ, Ortiz C. 2008. Cartilage aggrecan can undergo self-adhesion. Biophys. 7. 95:4862-70

146. Parker KH, Winlove CP, Maroudas A. 1988. The theoretical distributions and diffusivities of small ions in chondroitin sulphate and hyaluronate. Biophys. Chem. 32:271-82

147. de la Cruz MO, Belloni L, Delsanti M, Dalbiez JP, Spalla O, Drifford M. 1995. Precipitation of highly charged polyelectrolyte solutions in the presence of multivalent salts. F. Chem. Phys. 103:5781-91

148. MacGregor EA, Bowness JM. 1971. Interaction of proteoglycans and chondroitin sulfates with calcium or phosphate ions. Can. F. Biochem. 49:417-25 
149. Hunter GK, Wong KS, Kim JJ. 1988. Binding of calcium to glycosaminoglycans: an equilibrium dialysis study. Arch. Biochem. Biophys. 260:161-67

150. Lee H-Y, Kopesky PW, Plaas AHK, Sandy JD, Kisiday J, et al. 2010. Adult bone marrow stromal cell-based tissue-engineered aggrecan exhibits ultrastructure and nanomechanical properties superior to native cartilage. Osteoarthr. Cartil. 18:1477-86

151. Roughley PJ, White RJ. 1980. Age-related changes in the structure of the proteoglycan subunits from human articular cartilage. 7. Biol. Chem. 255:217-24

152. Inerot S, Heinegard D, Audell L, Olsson S-E. 1978. Articular-cartilage proteoglycans in aging and osteoarthritis. Biochem. F. 169:143-56

153. Raviv U, Giasson S, Kampf N, Gohy J-F, Jerome R, Klein J. 2003. Lubrication by charged polymers. Nature 425:163-65

154. Swann DA, Hendren RB, Radin EL, Sotman SL, Duda EA. 1981. The lubricating activity of synovial fluid glycoproteins. Arthritis Rheum. 24:22-30

155. Jay GD, Harris DA, Cha C-J. 2001. Boundary lubrication by lubricin is mediated by O-linked beta(13)Gal-GalNAc oligosaccharides. Glycoconj. f. 18:807-15

156. Jay GD, Tantravahi U, Britt DE, Barrach HJ, Cha C-J. 2001. Homology of lubricin and superficial zone protein (SZP): products of megakaryocyte stimulating factor (MSF) gene expression by human synovial fibroblasts and articular chondrocytes localized to chromosome 1q25. 7. Orthop. Res. 19:677-87

157. Briscoe WH, Titmuss S, Tiberg F, Thomas RK, McGillivray DJ, Klein J. 2006. Boundary lubrication under water. Nature 444:191-94

158. Hopwood JJ, Robinson HC. 1974. The structure and composition of cartilage keratan sulphate. Biochem. 7. $141: 517-26$

159. Marszalek PE, Li H, Oberhauser AF, FernandezJM. 2002. Chair-boat transitions in single polysaccharide molecules observed with force-ramp AFM. Proc. Natl. Acad. Sci. USA 99:4278-83

160. Scott JE. 2003. Elasticity in extracellular matrix "shape modules" of tendon, cartilage, etc. A sliding proteoglycan-filament model. 7. Physiol. 553:335-43

161. Merkel R, Nassoy P, Leung A, Ritchie K, Evans E. 1999. Energy landscapes of receptor-ligand bonds explored with dynamic force spectroscopy. Nature 397:50-53

162. Han L, Frank EH, Greene JJ, Lee H-Y, Hung H-HK, et al. 2011. Time-dependent nanomechanics of cartilage. Biophys. 7. 100(7):1846-54

163. Chang DP, Abu-Lail NI, Guilak F, Jay GD, Zauscher S. 2008. Conformational mechanics, adsorption, and normal force interactions of lubricin and hyaluronic acid on model surfaces. Langmuir 24:1183-93

164. Coles JM, Blum JJ, Jay GD, Darling EM, Guilak F, Zauscher S. 2008. In situ friction measurement on murine cartilage by atomic force microscopy. F. Biomech. 41:541-48

165. Liu X, Noble PC, Luo Z-P. 2004. Direct measurements of the compressive properties of single proteoglycan aggregates. Biochem. Biophys. Res. Commun. 316:313-16

166. Liu X, Sun JQ, Heggeness MH, Yeh M-L, Luo Z-P. 2004. Direct quantification of the rupture force of single hyaluronan/hyaluronan binding protein bonds. FEBS Lett. 563:23-27

167. Zhu Y, Granick S. 2003. Biolubrication: hyaluronic acid and the influence on its interfacial viscosity of an antiinflammatory drug. Macromolecules 36:973-76

168. Gleghorn JP, Bonassar LJ. 2008. Lubrication mode analysis of articular cartilage using Stribeck surfaces. 7. Biomech. 41:1910-18

169. Ateshian GA. 2009. The role of interstitial fluid pressurization in articular cartilage lubrication. F. Biomech. 42:1163-76

170. Schmidt TA, Sah RL. 2007. Effect of synovial fluid on boundary lubrication of articular cartilage. Osteoartbr. Cartil. 15:35-47

171. Cranford S, Buehler M. 2010. Coarse-graining parameterization and multiscale simulation of hierarchical systems. Part I. Theory and model formulation. In Multiscale Modeling: From Atoms to Devices, ed. P Derosa, T Cagin, pp. 13-34. Boca Raton, FL: CRC

172. Gong Y, Ogunniyi AO, Love JC. 2010. Massively parallel detection of gene expression in single cells using subnanolitre wells. Lab Chip 10:2334-37

173. Ogunniyi AO, Story CM, Papa E, Guillen E, Love JC. 2009. Screening individual hybridomas by microengraving to discover monoclonal antibodies. Nat. Protoc. 4:767-82 
174. Shan ZW, Mishra RK, Syed Asif SA, Warren OL, Minor AM. 2008. Mechanical annealing and sourcelimited deformation in submicrometre-diameter Ni crystals. Nat. Mater. 7:115-19

175. Browning ND, Campbell GH, Hawreliak JA, Kirk MA. 2010. In situ characterization of metals at extremes. MRS Bull. 35:1009-16

176. Edwards HK, Fay MW, Anderson SI, Scotchford CA, Grant DM, Brown PD. 2009. An appraisal of ultramicrotomy, FIBSEM and cryogenic FIBSEM techniques for the sectioning of biological cells on titanium substrates for TEM investigation. 7. Microsc. 234:16-25 


\section{Contents}

\section{Materials Science of Biological Systems}

Advances in Drug Delivery

Brian P. Timko, Kathryn Whitehead, Weizvei Gao, Daniel S. Kohane,

Omid Farokbzad, Daniel Anderson, and Robert Langer

Crystallization Pathways in Biomineralization

Steve Weiner and Lia Addadi

Deformation and Fracture Mechanisms of Bone and Nacre

Rizhi Wang and Himadri S. Gupta

Linear and Nonlinear Rheology of Living Cells

Philip Kollmannsberger and Ben Fabry

Mussel-Inspired Adhesives and Coatings

Bruce P. Lee, P.B. Messersmith, 7.N. Israelachvili, and 7.H. Waite

Nanomechanics of the Cartilage Extracellular Matrix

Lin Han, Alan 7. Grodzinsky, and Christine Ortiz

Plant Stems: Functional Design and Mechanics

Thomas Speck and Ingo Burgert

\section{Current Interest}

Elastic and Mechanical Properties of the MAX Phases

Michel W. Barsoum and Miladin Radovic

Electrocaloric Materials

F.F. Scott

Electrochromic Materials

Roger 7. Mortimer

Nanowire Solar Cells

Erik C. Garnett, Mark L. Brongersma, Yi Cui, and Michael D. McGehee

Nonconventional (Non-Silicon-Based) Photovoltaic Materials

T. Unold and H.W. Schock 
On the Future Availability of the Energy Metals

T.E. Graedel

Oxide Electronics Utilizing Ultrafast Metal-Insulator Transitions

Zheng Yang, Changhyun Ko, and Shriram Ramanathan

Point Defects in Oxides: Tailoring Materials Through

Defect Engineering

Harry L. Tuller and Sean R. Bishop

Recent Developments in Semiconductor Thermoelectric Physics and Materials

Ali Shakouri

Thermoelectric Phenomena, Materials, and Applications

Terry $M$. Tritt

Index

Cumulative Index of Contributing Authors, Volumes 37-41 449

\section{Errata}

An online log of corrections to Annual Review of Materials Research articles may be found at http://matsci.annualreviews.org/errata.shtml 